\title{
Subsieve Particle-Size Measurement of Metal Powders by Air Elutriation
}

\author{
Rolla E. Pollard
}

\begin{abstract}
Particle-size measurements of spherical metal powders by means of the Roller air analyzer, using samples up to 40 grams of powder, were found to be reproducible within approximately \pm 1 percent of the original weight of the sample for particle diameters up to 120 microns. The separation limits closely approximated those required by Stokes law $\left(V=K d^{2}\right)$ for particle diameters of 5 to 20 microns. For particle diameters of 20 to 70 microns, the size limits of fractions conformed approximately to the formula $V=K^{\prime \prime} d^{1.4}$. With particles larger than 70 microns, the accuracy of separation was uncertain.

Reproducible results also were obtained with irregularly shaped particles, such as those of electrolytic copper powder. However, the accuracy of calculated size limits was uncertain, no attempt being made to check them by other methods of measurement.
\end{abstract}

\section{Introduction}

Accurate and reproducible methods of determining particle size are of great importance in powder metallurgy. The size distribution of the particles markedly influences the inherent packing behavior of a powder. This vitally affects the pressing and sintering techniques employed and the properties of the sintered object produced.

Sieves are used almost universally for size analyses of particles large enough to be retained by a No. 325 sieve $(\approx-44 \pm 3$ microns).

For smaller, or subsieve, particles, other methods of size measurements must be used Among the many methods currently employed, air elutriation has a distinct advantage because dry, sized fractions can be collected and used for further study.

One of the few commercially available devices for determining particle-size distribution by air elutriation is the Reller air analyzer developed by P. S. Roller $[1,2,3,4]^{1}$ at the U. S. Bureau of Mines. Tentative standard methods of particle-size analysis based on this apparatus are being considered by the Metal Powder Association [5], and the American Society for Testing Materials [6]. The Roller air analyzer has been used at the National Bureau of Standards for several years in connection with studies of the properties of metal powders. An investigation was made to determine the effects of certain variations in equipment and operating technique on the reproducibility of the results obtained and the accuracy of the separations. These variations included time of testing or criterion of end point, condition of interior surface of the settling tubes, particle-size distribution of sample, range of particle sizes within a fraction, size of sample, humidity of air, and particle shape. Two prime objectives of the investigation were to establish the approximate range of application of Stokes law to metal-powder elutriation under the conditions found in the Roller analyzer, and to determine, if possible, the relation between the diameters and falling velocities for particles of larger size.

Figures in brackets indicate the literature references at the end of this paper*

\section{Theoretical Background}

Particle-size measurement by the Roller air analyzer is based on Stokes' law, according to which the terminal velocity of a particle falling freely in a homogeneous fluid is given by the formula

$$
V=K g \frac{\left(\rho-\rho_{0}\right) d^{2}}{\mu},
$$

where

$V=$ terminal velocity, $\mathrm{cm} / \mathrm{sec}$

$K=$ a constant that depends on the shape of the particle and the nature of the air flow

$g=$ gravitational constant $=980 \mathrm{~cm} / \mathrm{sec}^{2}$

$\rho=$ density of the particle, $\mathrm{g} / \mathrm{cm}^{3}$

$\rho_{0}=$ density of the fluid $=0.0012 \mathrm{~g} / \mathrm{cm}^{3}$ for air at $21^{\circ} \mathrm{C}$ and $760 \mathrm{~mm}$

$d=$ diameter of particle, $\mathrm{cm}$

$\mu=$ viscosity of the fluid $=1.82 \times 10^{-4}$ poise for air.

Stokes' law is strictly valid only for spherical particles and streamline flow. When $K=1 / 18$, then $V=g\left(\rho-\rho_{0}\right) d^{2} / 18 \mu$ or, with air as the fluid and $d$ expressed in microns, $V=0.00299\left(\rho-\rho_{0}\right) d^{2}$. Under nearly ideal conditions (that is, a single sphere fallingin a relatively large cylinder of quiescent fluid) the experimental measurement of the rate of fall of a sphere is a convenient method of determining the value of the viscosity of a fluid. Castleman [7] has. analyzed a number of such measurements to determine the range of Reynolds numbers ${ }^{2}$ over which Stokes' law gives accurate results. Castleman's mean curve coincides with the curve representingStokes' law at approximately $R=0.5$. For iron and copper powders in air, this would correspond to diameters of 32 and 31 microns, respectively.

However, such calculations assume ideal conditions not found in an air separator, such as the Roller, where large numbers of particles enter the settling

2 The Reynolds number, $R$, represents the ratio of the inertia terms to the frictional terms in the equations of motion of a fluid. In the case of a particle falling in a fluid, $R=d V \rho_{0} / \mu$. 
chambers simultaneously and the movement of individual particles may be influenced or interrupted by the presence of other particles or by the containing wall. The air also possesses motion, which changes in direction and in velocity while in contact with the particles and with the walls of the chamber. Moreover, most particles produced commercially are not true spheroids but are irregular in shape, thus further complicating such tests. In this respect Wadell [8] has found that with nonspherical particles consistent results can be obtained in sedimentation or ail elutriation tests only when the Reynolds number does not exceed $0.2(\mathrm{R}<0.2)$.

\section{Method and Materials}

The Roller air analyzer consists, essentially, of a device by which powder particles are blown into a settling chamber through which dried and metered air moves at a uniform measurable velocity. Means are provided for collecting the particles carried out of the settling chamber and for removing and recirculating lodged particles. A diagram of the apparatus used in this investigation is shown in figure 1. Low-pressure air is cleaned in the dust trap, $\mathrm{H}$, and the oil trap, G, before being dried in an activated alumina dryer, F. E is a vessel containing a moisture-indicating compound. Air flow is regulated by means of calibrated metering orifices, $\mathrm{A}$, and manometers, C and D. The air stream passing through the jet, J, at high velocity impinges on the powder sample, $K$. The $U$ tube, M, holding the sample is given a jerky, oscillatory motion by bumper arms rotating on the shaft, L. The oscillating motion of the $U$ tube (288 oscillations a $\mathrm{min}$ ) continually brings fresh material under the action of the jet and newly exposed powder particles of amenable sizes are blown into the settling tube, P. Theoretically, particles smaller than the selected size are carried out of the tube and collected in extraction thimble Q.

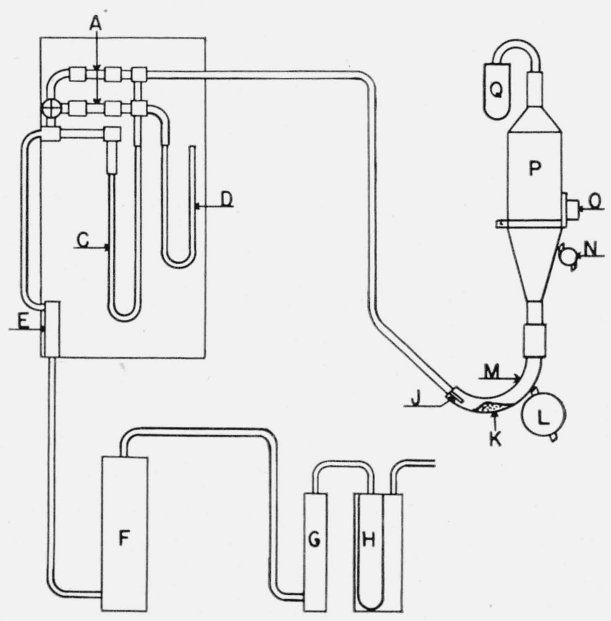

Figure 1. Details of Roller air analyzer assembly.
During the investigation it was found that alundum extraction thimbles, RA98 (coarse), were definitely superior to the paper thimbles originally used. For example, a paper thimble, during a 120 -min run with dried air, ${ }^{3}$ but without powders, will gain as much as $0.81 \mathrm{~g}$ in weight. This would amount to over 2 percent of the weight of a $40 \cdot \mathrm{g}$ sample. During this time an alundum thimble will gain only $0.004 \mathrm{~g}$. Moreover the alundum thimbles can be cleaned readily in nitric acid or aqua regia and reused.

Means are supplied for dislodging and recirculating material that sticks to the walls of the settling chamber by a tapping device, $\mathrm{N}$, with two leatherfaced hammers that strike the tube about 870 times a min. Later this device was supplemented by a Syntron vibrator, O, (type $\mathrm{V}-4,7,200$ vibrations a $\min$ ).

Four settling tubes with inside diameters of 9 , $4 \frac{1}{2}, 2 \frac{1}{4}$, and $1 \frac{11}{1} /$ in., respectively, were supplied with the apparatus. The three largest tubes vary in diameter by the ratio of $1 / 2$. Therefore, to the limit of application of Stokes' law $\left(V=K d^{2}\right)$ successive separations made in these tubes at the same rate of air flow would yield fractions wbose maximum particle diameter would vary in the same order by the ratio of 2. The same rate of air flow used in the 1/4-in. tube would give an air velocity lower than that calculated by Stokes' law by the ratio of $\left(1 \frac{1 / 8}{8}\right)^{2} /\left(1 \frac{1 / 4}{4}\right)^{2}$. Although it is not specifically mentioned by the manutacturer, it is possible that this change in ratic was intended as a correction for Stokes' law at high air velocities.

The metal powders used in the investigation are listed in table 1 . The permalloy powders, PC and $\mathrm{PF}$, were found to be predominantly spherical in shape, 90 to 95 percent of the particles having nearly perfectly circular projected images, as shown in figure 2. A mixture of the two powders (2 parts by weight of $\mathrm{PC}$ to 1 part of $\mathrm{PF}$, designated in table 1 as PM) covered the subsieve particle-size range fairly well and was used extensively in checking the accuracy of particle-size analyses by microscopic measurements. The atomized copper and zinc powders were also largely spherical in shape, as shown in figures 3 and 4, respectively. approximately 80 percent of the projected images of these two powders were either circular or slightly elliptical with ratios of minor to major axes of 0.9 or more. It was observed that there was not much difference in size distribution among the several fractions whether the perfectly circular images were measured or the minor axes of all elliptical images were included. The controlling dimension in the separation of these particles, therefore, seems to be the minor axis.

The other powders of table 1 were more or less irregular in shape. For example, the irregular shape of the electrolytic copper, $\mathrm{Cu} \mathrm{B}$, powder is shown in figure 5. No attempt was made to check the par-

3 The term "dried air" refers, in this text, to air dried in an activated alumina dryer to a nominal dewpoint range of $-40^{\circ}$ to $-70^{\circ} \mathrm{F}$. 


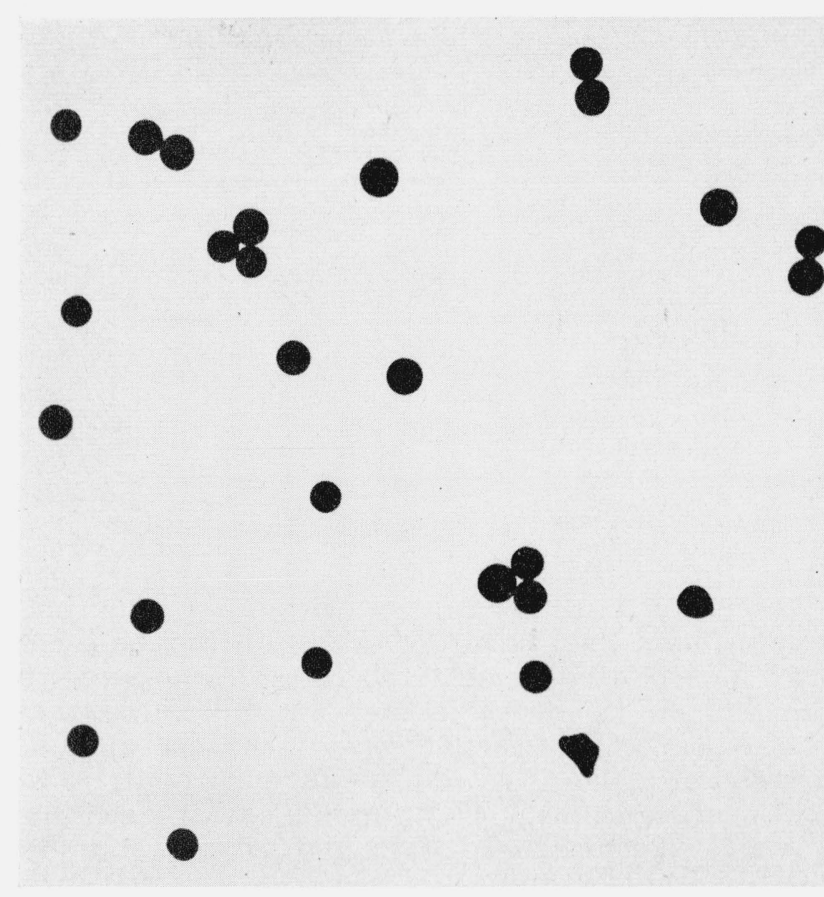

Figure 2. Almost perfectly spherical particles of +40 -micron
fraction of permalloy-mix powder Magnification $\times .100$.

-

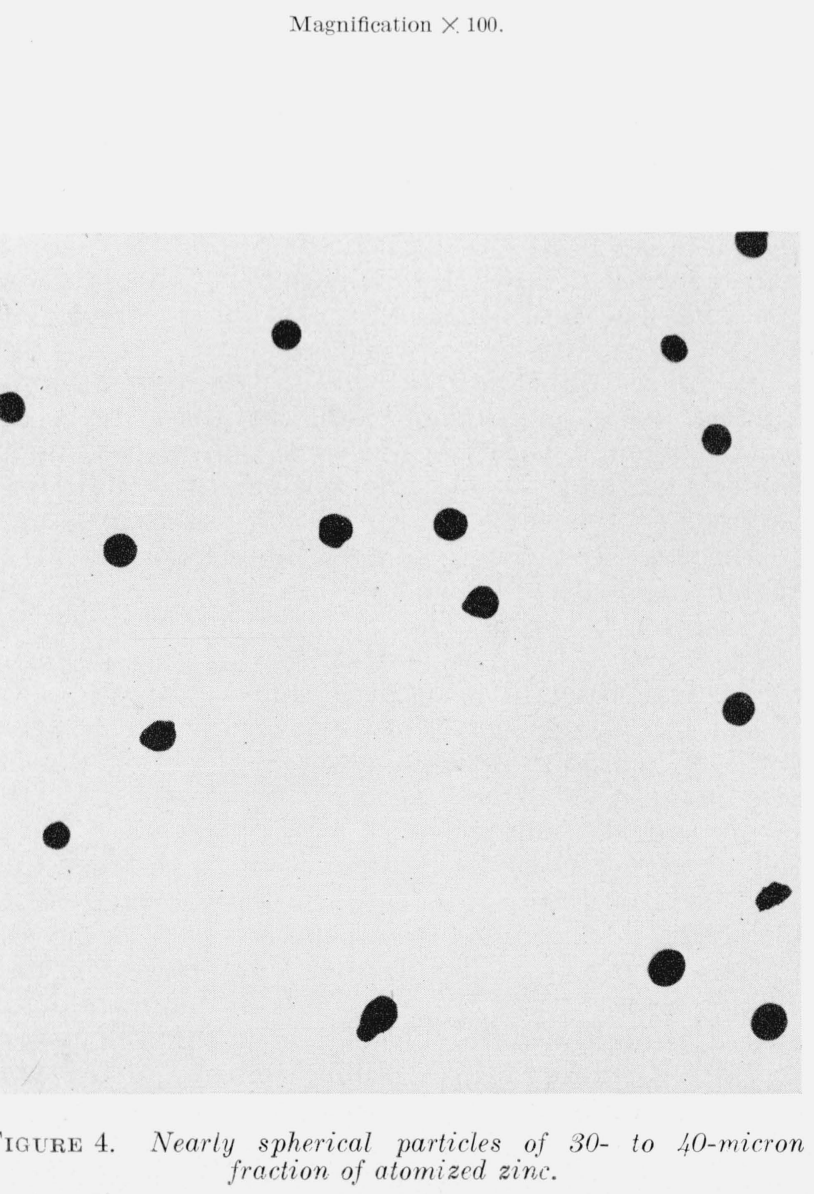

Magnification $\times 100$.
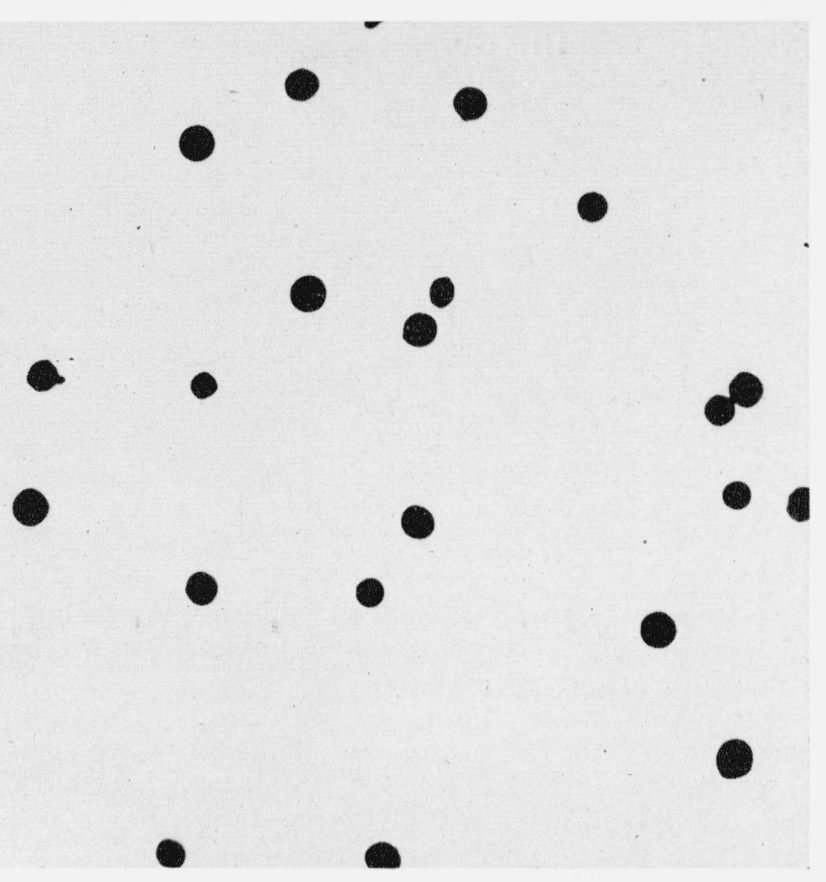

Figure 3. Nearly spherical particles of 30- to 40-micron fraction of atomized copper, $\mathrm{Cu} \mathrm{A}$, powder.

Magnification $\times 100$.
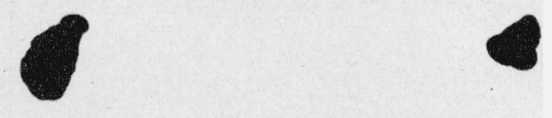

3
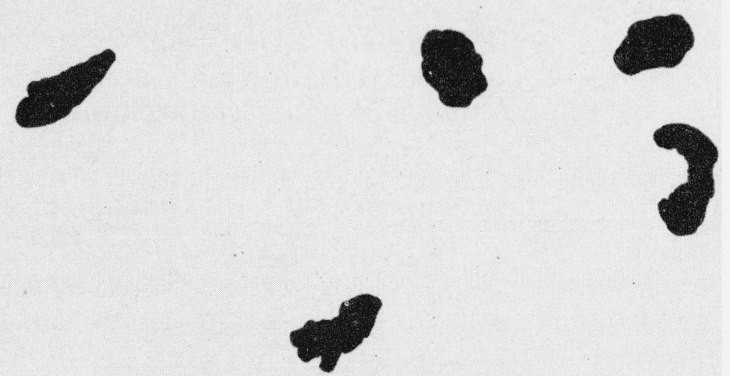

of electrolutic copper, $\mathrm{Cu}$ B, powder.

Magnification $\times 100$ 
TABLE 1. Description, designation, and pertinent properties of metal powders used in the investigation

\begin{tabular}{|c|c|c|c|c|c|c|c|}
\hline Powder & $\begin{array}{c}\text { Designation } \\
\text { in text }\end{array}$ & Source & Method of manufacture & Particle shape & Subsieve & $\begin{array}{l}\text { Apparent } \\
\text { density }\end{array}$ & $\begin{array}{l}\text { Theoret } \\
\text { ical solic } \\
\text { density }\end{array}$ \\
\hline $\begin{array}{l}\text { Permalloy, fine } \\
\text { Permalloy, coarse } \\
\text { Permalloy mix } \\
\text { Copper }\end{array}$ & 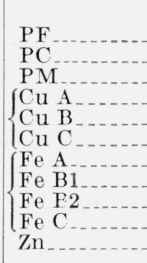 & $\begin{array}{l}\text { R-B-H Dispersions } \\
\text { do } \\
\text { New Jersey Zinc } \\
\text { Charles Hardy } \\
\text { do } \\
\text { Plastic metals } \\
\text { do do } \\
\text { General Aniline } \\
\text { Charles Hardy }\end{array}$ & \begin{tabular}{|c|} 
A tomization \\
do \\
do \\
Electrolytic- \\
do \\
do \\
Reduced mill scale_. \\
Carbonyl \\
A tomization \\
\end{tabular} & 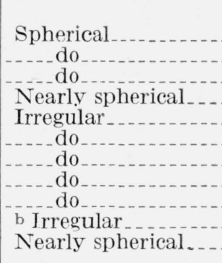 & $\begin{array}{l}\text { Percent } \\
100 \\
99.5+ \\
100- \\
46 \text { to } 60 \\
51 \text { to } 59 \\
90 \text { to } 95 \\
37 \text { to } 46 \\
32 \text { to } 40 \\
35 \text { to } 50 \\
99+ \\
99+\end{array}$ & $\begin{array}{l}\text { g/cm } \\
\text { 2. } 68 \\
4.40 \\
3.75 \\
\text { 5. } 26 \\
2.70 \\
1.61 \\
2.27 \\
2.52 \\
2.46 \\
3.10 \\
2.03\end{array}$ & $\begin{array}{l}\mathrm{g} / \mathrm{cm}^{3} \\
\text { a } 8.70 \\
\text { a } 8.70 \\
\text { a } 8.70 \\
8.96 \\
8.96 \\
8.96 \\
7.87 \\
7.87 \\
7.87 \\
7.87 \\
7.14\end{array}$ \\
\hline
\end{tabular}

a Estimated from the nominal composition; Ni, 78.5\%; Mo, 3.5\%; balance, Fe.

b Many individual particles of this powder ( $\mathrm{GAW}$. grade C) were spherical, but some of the spheres had grown together during production or sintered together during heat treatment, so that much of the final product was irregularly shaped.

ticle-size distribution of such powders by microscopic measurements. They were used, however, in studies involving reproducibility.

Attempts were made to determine the solid particle density of some of the powders used, according to ASTM D153-39, "Standard Method of Test for Specific Gravity of Pigments". The results were not very satisfactory, however, because the density of the kerosine changed during vacuum treatment to remove air occluded in the powder sample during its introduction into the kerosine. This change in density caused errors in the determination of the true volume of the sample. The range for permalloy powders, for example, was $8.70 \pm 0.06$ $\mathrm{g} / \mathrm{cm}^{3}$. This uncertainty undoubtedly could be reduced by employing suitable liquids of constant boiling point and possibly by using larger samples. It is believed, however, that the true solid density of all the powder particles used in this investigation, with the probable exception of the reduced iron powders $\mathrm{Fe} \mathrm{B}, \mathrm{Fe} \mathrm{B} 1$, and $\mathrm{Fe} \mathrm{B} 2$, are very close to the theoretical solid densities given in table 1 .

\section{Effects of Variations in Equipment and Testing Procedure on the Reproducibility of Results}

\subsection{Time of testing - criterion of end point}

Separation by air elutriation, like separation by sieving, is never complete. One of the first problems encountered in using the Roller air analyzer, therefore, is the adoption of some convention for establishing an artificial end point. The method originally used by Roller [1, 3, 4] was to operate by 30min intervals, weighing the amount collected for each interval. His stopping point, or artificial end point, was reached when the weight of powder collected in any interval was less than 10 percent of that collected in the first interval. This method also is recommended in a booklet published by the Metals Disintegrating Co. [9], and is being considered in the tentative standards of the MPA [5] and the ASTM [6]. The manufacturer, in the instructions sent with the apparatus, recommends a similar procedure but with 2 -min intervals.
Later Roller [10], working with some ceramic materials, found that when the weight of fraction separated was plotted against the rate of separation, a straight line or characteristic was obtained, that when extrapolated to zero rate, gave the total amount of material of the selected-size range originally present in the sample. The time intervals used for portland cement ranged from $1 / 2$ to $25 \mathrm{~min}$. Roller [11] has recently applied this end-point method to the particle-size analysis of metal powders, using time intervals as short as 1 or $2 \mathrm{~min}$, which, in the light of the results of the present investigation, appear to be of too short duration.

For example, a number of tests were made with the permalloy powder mix, PM, using time intervals of $2,4,8,15$, and $30 \mathrm{~min}$. Some of the tests were stopped after the interval in which the amount collected was less than 10 percent of that collected in the first interval (10-percent end point). Others were continued until the collection per interval was less than 1 percent of the first interval (1 percent end point).

In figure 6 the amounts of the 0 - to 10-micron fraction collected during some of these tests are plotted against the rate of separation, according to Roller's method $[10,11]$. It will be noted that those portions of the curves representing the early stages of the test (which were used by Roller) tend to flatten out with decreasing time intervals. In the 2 -min-interval curve, the 4 points representing the third, fourth, fifth, and sixth intervals may be fairly well represented by a straight line. Extrapolation of this line to zero rate, however, indicates a rather low result, $9.55 \mathrm{~g}$, or 21.4 percent, of a $40-\mathrm{g}$ sample as compared to $27 \pm 1$ percent as measured by the 10 -percent end point.

This may be due, at least in part, to the fact that for very short time intervals the largest particles of the selected size range do not have time to leave the settling chamber. The resultant velocity, $V_{R}$, of a particle in a settling tube depends in magnitude and direction on the difference between the velocity of the air, $V_{A}$, and the free-falling velocity of the particle, $V_{F}$. The $4 \frac{1}{2}$-in. settling tube, for example, has a section of uniform diameter about $28 \mathrm{~cm}$ in length. In order for a particle to escape from this tube $V_{R}$ must be greater than $28 / 60 t \mathrm{~cm} / \mathrm{sec}$, where 


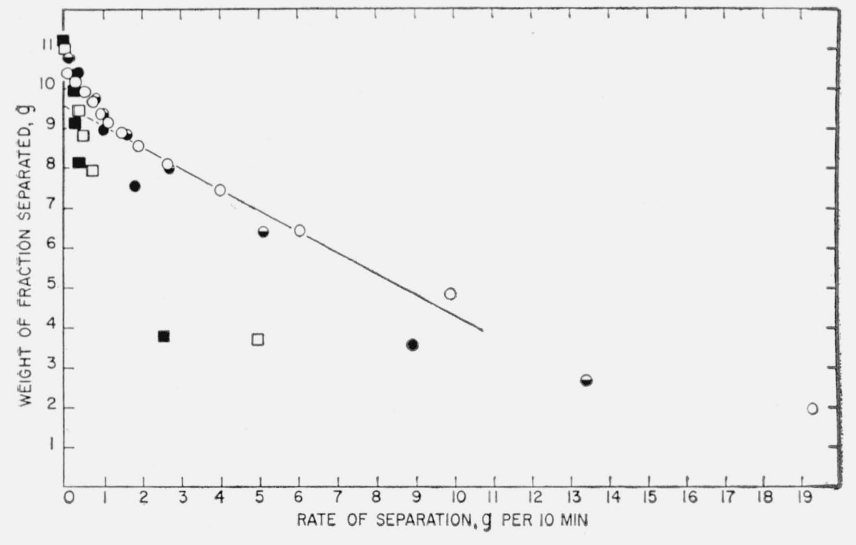

Figure 6. Roller's end-point method applied to 0 - to 10micron fraction of permalloy-mix powder.

Time intervals: $\bigcirc, 2 \mathrm{~min}$; $\bullet, 4 \mathrm{~min}$;, $8 \mathrm{~min}$; $\square, 15 \mathrm{~min}$;, $30 \mathrm{~min}$.

$t$ is the time interval in minutes. For a separation of permalloy powder $(\rho=8.7)$ with an air velocity calculated for a nominal particle diameter of 10 microns, it can be shown that the largest particle that could escape from the tube in a given time interval would be 9.1 microns for $1 \mathrm{~min}, 9.5$ for 2 min, and 9.97 for $30 \mathrm{~min}$. For very short time intervals, therefore, the actual separation would be at a diameter appreciably smaller than that for which the air velocity was calculated, and the results would be low, particularly if the size distribution of the sample were such that large numbers of borderline particles would be affected.

The influence of the time interval on the endpoint criterion is illustrated in figure 7 , which represents all of the tests made on the 0- to 10-micron fraction of the PM powder. The upper and lower solid curves represent, respectively, the highest and lowest values obtained with any time interval within the total testing time indicated on the scale of abscissas. The intermediate broken curves represent the lowest values obtained with 2-, 4-, 8- and 15 -min intervals. The total number of tests include: $2-, 4-$, and 8 -min intervals - 4 tests each, with 1 in each case carried to the 1-percent end point; 15 -min intervals -8 tests, with 3 - to the 1 -percent end point; 30 -min intervals - 67 tests, with 3 - to the 1-percent end point.

For tests with time intervals shorter than $30 \mathrm{~min}$, test conditions were uniform, except that in those continued to the 1-percent end point a Syntron vibrator, $\mathrm{O}$, figure 1 , was used in addition to the regular hammer, $\mathrm{N}$, figure 1 . The 30 -min-interval tests, however, included many in which the test conditions were varied considerably. For example, the three points just outside the "knee" of the upper curve (at 30,60, and $90 \mathrm{~min}$ ) were obtained without a vibrator but with humidified instead of dried air. Other tests were made with and without the vibrator and with samples varying in size from 20 to $100 \mathrm{~g}$.

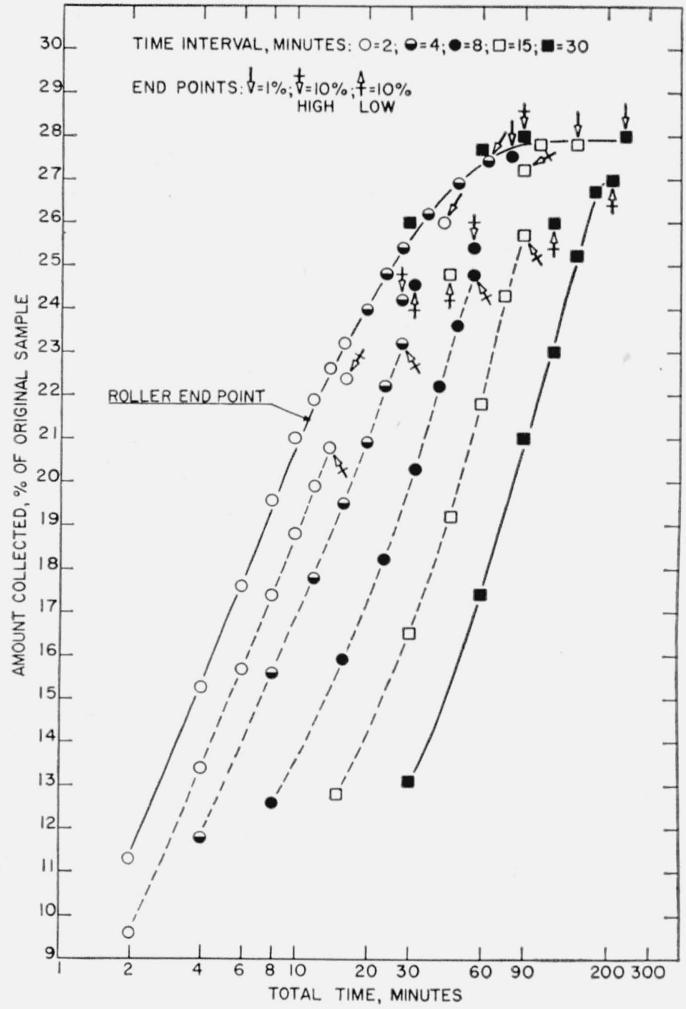

Fioure 7. Amount collected versus total time of testing for 0 - to 10-micron fractions of permalloy-mix powder with 2-, 4-, 8-, 15-, and 30-min intervals.

The solid curves represent the range of results obtained in a total of 90 tests. The broken curves represent the lowest values obtained with time intervals indicated by the symbols. The Roller end point is the end point determined in figure 6.

It will be noted that, for the 30-min interval, in spite of a very wide variation in initial separation rate (from 13 to 26 percent per interval), the results tended to converge so that the 10-percent end points (marked with barred arrows) varied no more than \pm 1 percent of the original weight of the sample. For the shorter time intervals the 10 -percent end points were progressively lower. These results show conclusively that to get within the end-point range of the 30 -min intervals, tests with shorter intervals would have to be carried beyond the 10-percent end point. All the 1-percent end points (marked with plain arrows) were within this range except the 2min interval, which was at the lower edge. Roller's end point (as determined in fig. 6) would be at 21.4 percent on the upper curve of figure 7 .

It was concluded from these results that $30-\mathrm{min}$ intervals and the 10-percent end-point criterion were most satisfactory. This procedure, therefore, was adopted as standard for all subsequent tests. Complete Roller analyses of permalloy and other metal powders are given in tables 2 to 9 inclusive. 
TABLE 2. Roller analyses of permalloy mix, PM, powder-40-g samples, minus No. 325 sieve, 30-min intervals, 10-percent end point, dry air

\begin{tabular}{|c|c|c|c|c|c|c|c|c|c|c|c|}
\hline \multirow{2}{*}{$\begin{array}{c}\text { Fraction } \\
\text { (microns) }\end{array}$} & \multirow{2}{*}{$\begin{array}{l}\text { Number } \\
\text { of tests }\end{array}$} & \multirow{2}{*}{$\begin{array}{l}\text { Diameter } \\
\text { of settling } \\
\text { tube }\end{array}$} & \multirow{2}{*}{$\begin{array}{l}\text { Stokes air } \\
\text { velocity }\end{array}$} & \multirow{2}{*}{ Air flow } & \multirow{2}{*}{$\begin{array}{l}\text { A verage air } \\
\text { velocity }\end{array}$} & \multicolumn{3}{|c|}{$\begin{array}{l}\text { Roller analysis-percentage by } \\
\text { weight of original sample }\end{array}$} & \multicolumn{3}{|c|}{$\begin{array}{l}\text { Typical microscopic count-percent- } \\
\text { age by weight of fraction }\end{array}$} \\
\hline & & & & & & Mean & $\begin{array}{l}\text { Maximum } \\
\text { deviation } \\
\text { from mean }\end{array}$ & $\begin{array}{l}\text { Standard } \\
\text { deviation }\end{array}$ & Undersize & Oversize & $\begin{array}{l}\text { Within } \\
\text { size } \\
\text { limits }\end{array}$ \\
\hline \multicolumn{12}{|c|}{ Series 1 - Tubes as received, hammer only, air velocity partly corrected a } \\
\hline $\begin{array}{r}0 \text { to } 5 \\
5 \text { to } 10 \\
10 \text { to } 20 \\
20 \text { to } 40 \\
+40 \\
\text { Recovery }\end{array}$ & $\begin{array}{l}4 \\
4 \\
4 \\
4 \\
4 \\
4 \\
4\end{array}$ & $\begin{array}{c}\text { Inches } \\
9 \\
41 / 2 \\
21 / 4 \\
11 / 4 \\
\\
\end{array}$ & $\begin{array}{c}\mathrm{cm} / \mathrm{sec} \\
0.65 \\
2.6 \\
10.4 \\
41.6 \\
-. .- \\
\end{array}$ & $\begin{array}{c}L / \min \\
16.0 \\
16.0 \\
16.0 \\
16.0 \\
- \\
-\end{array}$ & $\begin{array}{c}\mathrm{cm} / \mathrm{sec} \\
0.65 \\
2.6 \\
10.4 \\
33.7 \\
- \\
\end{array}$ & $\begin{array}{r}7.95 \\
11.75 \\
42.52 \\
27.40 \\
0.90 \\
90.50\end{array}$ & $\begin{array}{r}+5.1-2.2 \\
+8.2-6.7 \\
+0.4-0.9 \\
+.4-0.5 \\
+.1-0.1 \\
+6.9-9.0\end{array}$ & $\begin{array}{r}3.00 \\
5.36 \\
0.54 \\
.34 \\
.07 \\
6.53\end{array}$ & 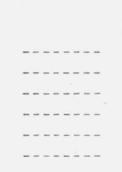 & 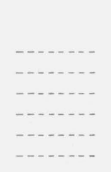 & 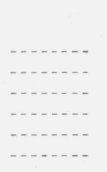 \\
\hline \multicolumn{12}{|c|}{ Series 2-Tubes as received, hammer only } \\
\hline $\begin{array}{r}0 \text { to } 20 \\
+20 \\
\text { Recovery }\end{array}$ & $\begin{array}{l}3 \\
3 \\
3\end{array}$ & $\begin{array}{c}21 / 4 \\
-\end{array}$ & $\begin{array}{c}10.4 \\
-\cdots\end{array}$ & $\begin{array}{c}16.0 \\
-\end{array}$ & $\begin{array}{c}10.4 \\
-\end{array}$ & $\begin{array}{l}68.9 \\
30.5 \\
99.4\end{array}$ & $\begin{array}{l}+1.5-2.3 \\
+2.3-1.3 \\
+0.2-0.2\end{array}$ & - & - & - & (n) \\
\hline \multicolumn{12}{|c|}{ Series 3-Tubes as received, hammer only, air velocity partly corrected a } \\
\hline $\begin{array}{r}0 \text { to } 10 \\
10 \text { to } 20 \\
20 \text { to } 30 \\
30 \text { to } 40 \\
+40 \\
\text { Recovery }\end{array}$ & $\begin{array}{rl}\mathrm{b} & 30 \\
\mathrm{~b} & 30 \\
8 \\
8 \\
8 \\
8\end{array}$ & $\begin{array}{c}41 / 2 \\
21 / 4 \\
11 / 4 \\
11 / 4 \\
--.- \\
\end{array}$ & $\begin{array}{r}2.6 \\
10.4 \\
23.4 \\
41.6 \\
-----. \\
--.-\end{array}$ & $\begin{array}{r}16.0 \\
16.0 \\
9.0 \\
16.0 \\
--.- \\
\end{array}$ & $\begin{array}{r}2.6 \\
10.4 \\
18.9 \\
33.7 \\
--- \\
\end{array}$ & $\begin{array}{r}27.14 \\
43.71 \\
18.11 \\
9.64 \\
0.94 \\
99.31\end{array}$ & $\begin{array}{r}+0.6-0.4 \\
+1.2-1.1 \\
+4.1-4.5 \\
+4.6-3.5 \\
+0.3-0.1 \\
+.4-0.5\end{array}$ & $\begin{array}{r}0.32 \\
.66 \\
3.13 \\
2.66 \\
0.16 \\
.32\end{array}$ & $\begin{array}{r}0 \\
0.6 \\
.3 \\
24.8 \\
5.8 \\
-.-\end{array}$ & $\begin{array}{l}0.7 \\
.9 \\
1.6 \\
4.7 \\
0\end{array}$ & $\begin{array}{l}99.3 \\
98.5 \\
98.1 \\
70.5 \\
94.2 \\
-\end{array}$ \\
\hline \multicolumn{12}{|c|}{ Series 4-Tubes as received, hammer and vibrator, air velocity partly corrected a } \\
\hline $\begin{array}{r}20 \text { to } 30 \\
30 \text { to } 40 \\
+40 \\
\text { Recovery }\end{array}$ & $\begin{array}{l}3 \\
3 \\
3 \\
3 \\
3 \\
3\end{array}$ & $\begin{array}{c}11 / 2 \\
11 / 4 \\
-.-1 \\
\end{array}$ & $\begin{array}{l}23.4 \\
41.6 \\
\end{array}$ & $\begin{array}{c}16.0 \\
16.0 \\
\end{array}$ & $\begin{array}{c}23.4 \\
33.7 \\
\\
\end{array}$ & $\begin{array}{r}23.03 \\
6.07 \\
0.70 \\
99.40\end{array}$ & $\begin{array}{r}+1.4-1.0 \\
+0.3-0.5 \\
+.1-0.1 \\
+.2-0.2\end{array}$ & $\begin{array}{r}1.02 \\
0.33 \\
.08 \\
.16\end{array}$ & $\begin{array}{l}0.2 \\
7.5 \\
5.3\end{array}$ & $\begin{array}{c}5.7 \\
10.1 \\
0\end{array}$ & $\begin{array}{r}94.1 \\
82.4 \\
94.7 \\
-\end{array}$ \\
\hline \multicolumn{12}{|c|}{ Series 5 - Tubes as received, hammer only, air velocity partly corrected a } \\
\hline $\begin{array}{r}20 \text { to } 30 \\
30 \text { to } 40 \\
+40 \\
\text { Recovery }\end{array}$ & $\begin{array}{l}4 \\
4 \\
4 \\
4\end{array}$ & \begin{tabular}{c}
$21 / 4$ \\
$11 / 4$ \\
\\
\hdashline
\end{tabular} & $\begin{array}{c}23.4 \\
41.6 \\
\end{array}$ & $\begin{array}{l}36.0 \\
16.0 \\
\\
\end{array}$ & $\begin{array}{c}23.4 \\
33.7 \\
\end{array}$ & $\begin{array}{r}27.15 \\
1.88 \\
0.82 \\
99.25\end{array}$ & $\begin{array}{l}+1.2-1.0 \\
+0.4-0.5 \\
+.1-0 \\
+.2-0.2\end{array}$ & $\begin{array}{r}0.79 \\
.35 \\
.04 \\
.15\end{array}$ & $\begin{array}{c}0.9 \\
.4 \\
\\
\end{array}$ & $\begin{array}{c}12.4 \\
11.6 \\
0\end{array}$ & $\begin{array}{c}86.7 \\
88.0 \\
100 \\
\end{array}$ \\
\hline \multicolumn{12}{|c|}{ Series 6-Small tubes lapped, vibrator and hammer, air velocity partly corrected a } \\
\hline $\begin{array}{r}20 \text { to } 30 \\
30 \text { to } 40 \\
+40 \\
\text { Recovery }\end{array}$ & $\begin{array}{l}10 \\
10 \\
10 \\
10\end{array}$ & $\begin{array}{c}11 / 2 \\
11 / 4 \\
-\end{array}$ & $\begin{array}{c}23.4 \\
41.6 \\
-\end{array}$ & $\begin{array}{c}16.0 \\
16.0 \\
\end{array}$ & $\begin{array}{r}23.4 \\
33.7 \\
\end{array}$ & $\begin{array}{r}24.50 \\
2.70 \\
0.85 \\
99.34\end{array}$ & $\begin{array}{r}+1.2-0.6 \\
+0.3-0.3 \\
+.3-0.2 \\
+.4-0.7\end{array}$ & $\begin{array}{l}0.50 \\
.18 \\
.16 \\
.36\end{array}$ & $\begin{array}{l}0.1 \\
0 \\
.4\end{array}$ & $\begin{array}{c}16.3 \\
20.8 \\
0\end{array}$ & $\begin{array}{l}83.6 \\
79.2 \\
99.6\end{array}$ \\
\hline \multicolumn{12}{|c|}{ Series 7-Small tubes lapped, vibrator and hammer, air velocity corrected ( $V=K^{\prime \prime} d^{14}$ above 20 microns) } \\
\hline $\begin{array}{r}20 \text { to } 30 \\
30 \text { to } 40 \\
+40 \\
\text { Recovery }\end{array}$ & $\begin{array}{l}5 \\
5 \\
5 \\
5\end{array}$ & $\begin{array}{l}11 / 2 \\
11 / 4 \\
0\end{array}$ & $\begin{array}{l}23.4 \\
41.6 \\
\end{array}$ & $\begin{array}{c}12.9 \\
13.3 \\
\\
\end{array}$ & $\begin{array}{l}18.7 \\
27.9 \\
\end{array}$ & $\begin{array}{r}21.35 \\
4.54 \\
1.82 \\
99.16\end{array}$ & $\begin{array}{r}+0.5-0.5 \\
+.3-0.2 \\
+.5-0.3 \\
+.3-0.4\end{array}$ & $\begin{array}{l}0.33 \\
0.18 \\
0.19 \\
0.26\end{array}$ & $\begin{array}{r}0.6 \\
.6 \\
.3 \\
-. .2\end{array}$ & $\begin{array}{l}1.3 \\
1.2 \\
0\end{array}$ & $\begin{array}{l}98.1 \\
98.2 \\
99.7 \\
\end{array}$ \\
\hline
\end{tabular}

${ }^{a}$ Air flow corresponding to Stokes air velocity used in all tubes except $11 \frac{1}{4}$ in., for which the air flow was calculated for 118 -in. diameter.

b These 30 tests include the 0 - to 10 - and 10 - to 20 -micron fractions of series $3,4,5,6$, and 7 . 
TABLE 3. Roller analyses of atomized copper, Cu A, and electrolytic copper, Cu B, powders, minus No. 325 sieve, 30-min intervals, 10-percent end point, dry air

\begin{tabular}{|c|c|c|c|c|c|c|c|c|c|c|c|}
\hline \multirow{2}{*}{$\begin{array}{l}\text { Fraction } \\
\text { (microns) }\end{array}$} & \multirow{2}{*}{$\begin{array}{l}\text { Number } \\
\text { of tests }\end{array}$} & \multirow{2}{*}{$\begin{array}{l}\text { Diameter } \\
\text { of settling } \\
\text { tube }\end{array}$} & \multirow{2}{*}{$\begin{array}{l}\text { Stokes air } \\
\text { velocity }\end{array}$} & \multirow{2}{*}{ Air flow } & \multirow{2}{*}{$\begin{array}{l}\text { A verage air } \\
\text { velocity }\end{array}$} & \multicolumn{3}{|c|}{$\begin{array}{l}\text { Roller analysis-percentage by } \\
\text { weight of original sample }\end{array}$} & \multicolumn{3}{|c|}{$\begin{array}{l}\text { Typical microscopic count-percent- } \\
\text { age by weight of fraction }\end{array}$} \\
\hline & & & & & & Mean & $\begin{array}{l}\text { Maximum } \\
\text { deviation } \\
\text { from mean }\end{array}$ & $\begin{array}{l}\text { Standard } \\
\text { deviation }\end{array}$ & Undersize & Oversize & $\begin{array}{l}\text { Within } \\
\text { size } \\
\text { limits }\end{array}$ \\
\hline \multicolumn{12}{|c|}{ Series 1 - Cu B-25-g samples, tubes as received, hammer only, air flow partly corrected a } \\
\hline $\begin{array}{r}0 \text { to } 5 \\
5 \text { to } 10 \\
10 \text { to } 20 \\
20 \text { to } 40 \\
+40 \\
\text { Recovery }\end{array}$ & $\begin{array}{l}14 \\
14 \\
14 \\
14 \\
14 \\
14\end{array}$ & $\begin{array}{c}\text { Inches } \\
9 \\
41 / 2 \\
21 / 4 \\
11 / 4 \\
\\
\end{array}$ & $\begin{array}{c}\mathrm{cm} / \mathrm{sec} \\
0.67 \\
2.7 \\
10.7 \\
42.9 \\
\end{array}$ & $\begin{array}{c}L / \min \\
16.5 \\
16.5 \\
16.5 \\
16.5 \\
\end{array}$ & $\begin{array}{c}\mathrm{cm} / \mathrm{sec} \\
0.67 \\
2.7 \\
10.7 \\
34.7 \\
\end{array}$ & $\begin{array}{r}4.92 \\
16.04 \\
32.46 \\
37.41 \\
7.42 \\
98.19\end{array}$ & $\begin{array}{r}+0.8-1.3 \\
+.9-1.0 \\
+1.4-1.6 \\
+1.5-2.8 \\
+2.3-3.9 \\
+1.6-2.0\end{array}$ & $\begin{array}{r}0.53 \\
.56 \\
.81 \\
1.11 \\
1.81 \\
1.13\end{array}$ & - & - & - \\
\hline \multicolumn{12}{|c|}{ Series $2-\mathrm{Cu}$ B-25-g samples, tubes as received, hammer only } \\
\hline $\begin{array}{r}0 \text { to } 20 \\
+20 \\
\text { Recovery }\end{array}$ & $\begin{array}{l}3 \\
3 \\
3\end{array}$ & $41 / 2$ & 10.7 & $\begin{array}{c}16.5 \\
-. .\end{array}$ & 10.7 & $\begin{array}{l}49.6 \\
50.0 \\
99.6\end{array}$ & $\begin{array}{l}+1.1-1.3 \\
+1.2-1.1 \\
+0-0\end{array}$ & n. & - & $\cdots$ & - \\
\hline \multicolumn{12}{|c|}{ Series 3-Cu A-40-g samples, tubes as received, hammer only, air velocity partly corrected a } \\
\hline $\begin{array}{r}0 \text { to } 5 \\
5 \text { to } 10 \\
10 \text { to } 20 \\
20 \text { to } 40 \\
+40 \\
\text { Recovery }\end{array}$ & $\begin{array}{l}5 \\
5 \\
5 \\
5 \\
5 \\
5\end{array}$ & $\begin{array}{l}9 \\
41 / 2 \\
21 / 4 \\
11 / 4 \\
\\
\cdots\end{array}$ & $\begin{array}{c}0.67 \\
2.7 \\
10.7 \\
42.9 \\
\end{array}$ & $\begin{array}{l}16.5 \\
16.5 \\
16.5 \\
16.5 \\
\cdots\end{array}$ & $\begin{array}{c}0.67 \\
2.7 \\
10.7 \\
34.7 \\
\end{array}$ & $\begin{array}{r}0.98 \\
3.94 \\
19.30 \\
44.28 \\
30.12 \\
98.40\end{array}$ & $\begin{array}{r}+0.1-0.1 \\
+.3-0.2 \\
+.7-0.5 \\
+3.1-3.0 \\
+4.5-4.6 \\
+1.3-0.5\end{array}$ & $\begin{array}{r}0.08 \\
.19 \\
.57 \\
2.54 \\
3.71 \\
0.71\end{array}$ & - & . & - \\
\hline \multicolumn{12}{|c|}{ Series 4 - Cu A-40-g samples, tubes as received, vibrator and hammer, air velocity partly corrected a } \\
\hline $\begin{array}{r}0 \text { to } 10 \\
10 \text { to } 20 \\
20 \text { to } 30 \\
30 \text { to } 40 \\
+40 \\
\text { Recovery }\end{array}$ & $\begin{array}{l}8 \\
8 \\
8 \\
8 \\
8 \\
8\end{array}$ & $\begin{array}{l}41 / 2 \\
21 / 4 \\
11 / 2 \\
11 / 4 \\
\end{array}$ & $\begin{array}{l}2.7 \\
10.7 \\
24.1 \\
42.9 \\
\end{array}$ & $\begin{array}{l}16.5 \\
16.5 \\
16.5 \\
16.5 \\
-. .-\end{array}$ & $\begin{array}{r}2.7 \\
10.7 \\
24.1 \\
34.7 \\
\end{array}$ & $\begin{array}{r}5.55 \\
18.06 \\
26.18 \\
18.30 \\
31.78 \\
99.71\end{array}$ & $\begin{array}{r}+0.1-0.4 \\
+.6-0.9 \\
+1.1-0.5 \\
+1.4-1.8 \\
+1.4-1.8 \\
+0.3-0.5\end{array}$ & $\begin{array}{l}0.16 \\
.38 \\
.53 \\
1.18 \\
1.02 \\
0.24\end{array}$ & $\begin{array}{l}0 \\
0 \\
0.3 \\
0 \\
0\end{array}$ & $\begin{array}{c}2.3 \\
1.8 \\
11.3 \\
10.9 \\
0\end{array}$ & $\begin{array}{l}97.7 \\
98.2 \\
88.4 \\
88.2 \\
100\end{array}$ \\
\hline \multicolumn{12}{|c|}{ Series 5-Cu A-40-g samples, small tubes lapped, vibrator and hammer, air velocity corrected $\left(V=K^{\prime \prime} d^{1.4}\right.$ above 20 microns) } \\
\hline $\begin{array}{r}0 \text { to } 10 \\
10 \text { to } 20 \\
20 \text { to } 30 \\
30 \text { to } 40 \\
+40 \\
\text { Recovery }\end{array}$ & $\begin{array}{l}2 \\
2 \\
2 \\
2 \\
2 \\
2 \\
2\end{array}$ & $\begin{array}{c}41 / 2 \\
21 / 4 \\
11 / 2 \\
11 / 4 \\
\\
\end{array}$ & $\begin{array}{c}2.7 \\
10.7 \\
24.1 \\
42.9 \\
-\end{array}$ & $\begin{array}{l}16.5 \\
16.5 \\
13.1 \\
13.6 \\
\\
\end{array}$ & $\begin{array}{c}2.7 \\
10.7 \\
19.1 \\
28.6 \\
\cdots\end{array}$ & $\begin{array}{r}5.2 \\
17.0 \\
14.5 \\
13.4 \\
50.0 \\
99.9\end{array}$ & $\begin{array}{r}+0.1-0.1 \\
+.1-0.2 \\
+.4-0.4 \\
+.6-0.7 \\
+.3-0.4 \\
+.1-0.1\end{array}$ & n...... & $\begin{array}{r}0 \\
0.1 \\
.1 \\
.8 \\
2.2 \\
\end{array}$ & $\begin{array}{l}1.3 \\
1.8 \\
3.2 \\
1.9 \\
0\end{array}$ & $\begin{array}{l}98.7 \\
98.1 \\
96.8 \\
97.3 \\
97.8 \\
\end{array}$ \\
\hline
\end{tabular}

a Air flow corresponding to Stokes air velocity used in all tubes except 11/4 in., for which the air flow was calculated for 11/8-in. diameter. 
TABLE 4. Roller analyses of hydrogen reduced iron powders, Fe B1 and Fe B2, 40-g samples, minus No. 325 sieve 30-min intervals, 10-percent end point, dry air

\begin{tabular}{|c|c|c|c|c|c|c|c|c|}
\hline \multirow{2}{*}{$\begin{array}{l}\text { Fraction } \\
\text { (microns) }\end{array}$} & \multirow{2}{*}{$\begin{array}{l}\text { Number } \\
\text { of tests }\end{array}$} & \multirow{2}{*}{$\begin{array}{l}\text { Diameter } \\
\text { of settling } \\
\text { tube }\end{array}$} & \multirow{2}{*}{$\begin{array}{l}\text { Stokes air } \\
\text { velocity }\end{array}$} & \multirow{2}{*}{ Air flow } & \multirow{2}{*}{$\begin{array}{l}\text { A verage } \\
\text { Air } \\
\text { velocity }\end{array}$} & \multicolumn{3}{|c|}{$\begin{array}{l}\text { Roller analysis-percentage by weight } \\
\text { of original sample }\end{array}$} \\
\hline & & & & & & Mean & $\begin{array}{l}\text { Maximum } \\
\text { deviation } \\
\text { from mean }\end{array}$ & $\begin{array}{l}\text { Standard } \\
\text { deviation }\end{array}$ \\
\hline \multicolumn{9}{|c|}{ Series $1-\mathrm{Fe}$ B1-Tubes as received, hammer only, air flow partly corrected a } \\
\hline $\begin{array}{c}0 \text { to } 5 \\
5 \text { to } 10 \\
10 \text { to } 20 \\
20 \text { to } 40 \\
+40 \\
\text { Recovery }\end{array}$ & $\begin{array}{l}23 \\
23 \\
23 \\
23 \\
23 \\
23\end{array}$ & $\begin{array}{c}\text { Inches } \\
9 \\
41 / 2 \\
21 / 4 \\
11 / 4 \\
\end{array}$ & $\begin{array}{c}\mathrm{cm} / \mathrm{sec} \\
0.6 \\
2.4 \\
9.4 \\
37.6 \\
-\end{array}$ & $\begin{array}{c}L / \min \\
14.5 \\
14.5 \\
14.5 \\
14.5 \\
\end{array}$ & $\begin{array}{c}\mathrm{cm} / \mathrm{sec} \\
0.6 \\
2.4 \\
9.4 \\
30.5 \\
\\
-.-\end{array}$ & $\begin{array}{r}1.67 \\
1.64 \\
14.69 \\
63.92 \\
15.70 \\
97.62\end{array}$ & $\begin{array}{r}+0.4-0.7 \\
+.6-0.5 \\
+.8-1.0 \\
+5.1-5.2 \\
+5.7-4.5 \\
+1.8-6.0\end{array}$ & $\begin{array}{r}0.28 \\
.32 \\
.57 \\
2.90 \\
2.84 \\
1.67\end{array}$ \\
\hline \multicolumn{9}{|c|}{ Series 2-Fe B2-Small tubes lapped, vibrator and hammer, air flow partly corrected a } \\
\hline $\begin{array}{r}0 \text { to } 10 \\
10 \text { to } 20 \\
20 \text { to } 30 \\
30 \text { to } 40 \\
+40 \\
\text { Recovery }\end{array}$ & $\begin{aligned} & \text { b } 11 \\
& \text { b } 11 \\
& 6 \\
& \\
& 6 \\
& \\
& \quad 6 \\
& 6\end{aligned}$ & $\begin{array}{l}41 / 2 \\
21 / 4 \\
11 / 2 \\
11 / 4 \\
-\cdot-.-- \\
\end{array}$ & $\begin{array}{r}2.4 \\
9.4 \\
21.2 \\
37.6 \\
--- \\
\end{array}$ & $\begin{array}{r}14.5 \\
14.5 \\
14.5 \\
14.5 \\
\end{array}$ & $\begin{array}{r}0.6 \\
2.4 \\
21.2 \\
30.5 \\
--.-\end{array}$ & $\begin{array}{r}4.47 \\
17.74 \\
36.45 \\
33.70 \\
7.50 \\
99.76\end{array}$ & $\begin{array}{r}+0.3-0.3 \\
+.4-0.3 \\
+.7-1.8 \\
+1.1-1.3 \\
+0.9-1.1 \\
+.1-0.3\end{array}$ & $\begin{array}{l}0.15 \\
.27 \\
.86 \\
.83 \\
.80 \\
.15\end{array}$ \\
\hline \multicolumn{9}{|c|}{ Series 3-Fe B2-Small tubes lapped, vibrator and hammer, air flow corrected ( $V=K^{\prime \prime} d^{1.4}$ above 20 microns) } \\
\hline $\begin{array}{r}20 \text { to } 30 \\
30 \text { to } 40 \\
\quad+40 \\
\text { Recovery }\end{array}$ & $\begin{array}{l}5 \\
5 \\
5 \\
5\end{array}$ & $\begin{array}{c}11 / 2 \\
11 / 4 \\
\\
\end{array}$ & $\begin{array}{c}21.2 \\
37.6 \\
-\end{array}$ & $\begin{array}{l}11.8 \\
12.2 \\
\end{array}$ & \begin{tabular}{l}
17.2 \\
25.8 \\
\hdashline- \\
\end{tabular} & $\begin{array}{l}26.00 \\
32.38 \\
18.70 \\
99.46\end{array}$ & $\begin{array}{r}+1.0-0.8 \\
+0.8-1.1 \\
+.9-1.4 \\
+.3-0.6\end{array}$ & $\begin{array}{r}0.75 \\
.74 \\
.86 \\
.31\end{array}$ \\
\hline
\end{tabular}

a Air flow corresponding to Stokes air velocity used in all tubes except 11/4 in., for which the air flow was calculated for 11/8-in. diameter. b These tests include the 0 - to 10 - and 10 - to 20 -micron fractions of series 2 and 3 .

TABLE 5. Roller-analyses of electrolytic iron powder, Fe A, 40-g samples, minus No. 325 sieve, 30-min intervals, 10-percent end point, dry air

\begin{tabular}{|c|c|c|c|c|c|c|c|c|}
\hline \multirow{2}{*}{$\begin{array}{l}\text { Fraction } \\
\text { (microns) }\end{array}$} & \multirow{2}{*}{$\begin{array}{l}\text { Number } \\
\text { of tests }\end{array}$} & \multirow{2}{*}{$\begin{array}{c}\text { Diameter } \\
\text { of settling } \\
\text { tube }\end{array}$} & \multirow{2}{*}{$\begin{array}{l}\text { Stokes air } \\
\text { velocity }\end{array}$} & \multirow{2}{*}{ Air flow } & \multirow{2}{*}{$\begin{array}{l}\text { A verage } \\
\text { A ir } \\
\text { velocity }\end{array}$} & \multicolumn{3}{|c|}{$\begin{array}{l}\text { Roller analysis - percentage by weight } \\
\text { of original fraction }\end{array}$} \\
\hline & & & & & & Mean & $\begin{array}{l}\text { Maximum } \\
\text { deviation } \\
\text { from mean }\end{array}$ & $\begin{array}{l}\text { Standard } \\
\text { deviation }\end{array}$ \\
\hline \multicolumn{9}{|c|}{ Series 1-Tubes as received, hammer only, air flow partly corrected a } \\
\hline $\begin{array}{r}0 \text { to } 5 \\
5 \text { to } 10 \\
10 \text { to } 20 \\
20 \text { to } 40 \\
+40 \\
\text { Recovery }\end{array}$ & $\begin{array}{l}12 \\
12 \\
12 \\
12 \\
12 \\
12\end{array}$ & $\begin{array}{l}\text { Inches } \\
9 \\
41 / 2 \\
21 / 4 \\
11 / 4 \\
-.-- \\
\end{array}$ & $\begin{array}{c}\mathrm{cm} / \mathrm{sec} \\
0.6 \\
2.4 \\
9.4 \\
37.6 \\
---. \\
--.-\end{array}$ & $\begin{array}{c}L / \min \\
14.5 \\
14.5 \\
14.5 \\
14.5 \\
\end{array}$ & $\begin{array}{c}\mathrm{cm} / \mathrm{sec} \\
0.6 \\
2.4 \\
9.4 \\
30.5 \\
-\end{array}$ & $\begin{array}{r}1.66 \\
5.77 \\
23.73 \\
61.89 \\
5.58 \\
98.48\end{array}$ & $\begin{array}{r}+0.2-0.3 \\
+.4-0.5 \\
+.8-0.4 \\
+1.3-0.7 \\
+1.1-1.6 \\
+0.8-1.2\end{array}$ & $\begin{array}{l}0.14 \\
.31 \\
.44 \\
.59 \\
.72 \\
.63\end{array}$ \\
\hline \multicolumn{9}{|c|}{ Series 2-Small tubes lapped, hammer and vibrator, air flow partly corrected a } \\
\hline $\begin{array}{r}0 \text { to } 10 \\
10 \text { to } 20 \\
20 \text { to } 30 \\
30 \text { to } 40 \\
+40 \\
\text { Recovery }\end{array}$ & $\begin{aligned} \text { b } 11 \\
\text { b } 11 \\
5 \\
5 \\
5 \\
5\end{aligned}$ & $\begin{array}{l}41 / 2 \\
21 / 4 \\
11 / 2 \\
11 / 4\end{array}$ & $\begin{array}{r}2.4 \\
9.4 \\
21.2 \\
37.6 \\
-\cdots\end{array}$ & $\begin{array}{l}14.5 \\
14.5 \\
14.5 \\
14.5 \\
- \\
-\end{array}$ & $\begin{array}{r}2.4 \\
9.4 \\
21.2 \\
30.5 \\
---.--\end{array}$ & $\begin{array}{r}6.30 \\
23.18 \\
38.36 \\
24.06 \\
6.78 \\
99.54\end{array}$ & $\begin{array}{r}+0.2-0.4 \\
+1.1-1.4 \\
+1.0-0.8 \\
+1.3-0.8 \\
+0.7-0.7 \\
+.3-0.2\end{array}$ & $\begin{array}{l}0.18 \\
.86 \\
.82 \\
.93 \\
.58 \\
.16\end{array}$ \\
\hline \multicolumn{9}{|c|}{ Series 3-Small tubes lapped, hammer and vibrator, air flow corrected ( $V=K^{\prime \prime} d^{1.4}$ above 20 microns) } \\
\hline $\begin{array}{r}20 \text { to } 30 \\
30 \text { to } 40 \\
+40 \\
\text { Recovery }\end{array}$ & $\begin{array}{l}6 \\
6 \\
6 \\
6\end{array}$ & $\begin{array}{l}11 / 2 \\
11 / 4 \\
\end{array}$ & \begin{tabular}{l}
21.2 \\
37.6 \\
\hdashline..--
\end{tabular} & $\begin{array}{l}11.8 \\
12.2 \\
\end{array}$ & $\begin{array}{c}17.2 \\
25.8 \\
\end{array}$ & $\begin{array}{l}25.37 \\
23.97 \\
21.60 \\
99.82\end{array}$ & $\begin{array}{r}+0.5-0.7 \\
+.9-1.2 \\
+.6-1.5 \\
+.2-0.4\end{array}$ & $\begin{array}{l}0.45 \\
.88 \\
.75 \\
.17\end{array}$ \\
\hline
\end{tabular}

a Air flow corresponding to Stokes air velocity used in all tubes except 11/4 in., for which the air flow was calculated for 11/8-in, diameter. b These tests include the 0 - to 10 - and 10 - to 20 -micron fractions of series 2 and 3 . 
TABLE 6. Roller analyses of carbonyl iron, Fe C, and electrolytic copper, Cu C, powders, minus No. 325 sieve, 30-min intervals' 10-percent end point, dry air

\begin{tabular}{|c|c|c|c|c|c|c|c|c|}
\hline \multirow{2}{*}{$\begin{array}{l}\text { Fraction } \\
\text { (microns) }\end{array}$} & \multirow{2}{*}{$\begin{array}{l}\text { Number } \\
\text { of tests }\end{array}$} & \multirow{2}{*}{$\begin{array}{l}\text { Diameter } \\
\text { of settling } \\
\text { tube }\end{array}$} & \multirow{2}{*}{$\begin{array}{l}\text { Stokes air } \\
\text { velocity }\end{array}$} & \multirow{2}{*}{ Air flow } & \multirow{2}{*}{$\begin{array}{l}\text { A verage } \\
\text { Air } \\
\text { velocity }\end{array}$} & \multicolumn{3}{|c|}{$\begin{array}{l}\text { Roller analysis-percentage by weight } \\
\text { of original sample }\end{array}$} \\
\hline & & & & & & Mean & $\begin{array}{l}\text { Maximum } \\
\text { deviation } \\
\text { from mean }\end{array}$ & $\begin{array}{l}\text { Standard } \\
\text { deviation }\end{array}$ \\
\hline \multicolumn{9}{|c|}{ Series 1-Fe C, 30-g samples, tubes as received, hammer only } \\
\hline $\begin{array}{r}0 \text { to } 5 \\
5 \text { to } 10 \\
10 \text { to } 20 \\
+20 \\
\text { Recovery }\end{array}$ & $\begin{array}{l}14 \\
14 \\
14 \\
14 \\
14 \\
14\end{array}$ & $\begin{array}{c}\text { Inches } \\
9 \\
41 / 2 \\
21 / 4 \\
\\
\end{array}$ & $\begin{array}{c}\mathrm{cm} / \mathrm{sec} \\
0.6 \\
2.4 \\
9.4 \\
\end{array}$ & $\begin{array}{c}L / \min \\
14.5 \\
14.5 \\
14.5 \\
\end{array}$ & $\begin{array}{c}\mathrm{cm} / \mathrm{sec} \\
0.6 \\
2.4 \\
9.4 \\
\end{array}$ & $\begin{array}{r}27.73 \\
53.07 \\
15.87 \\
0.92 \\
97.61\end{array}$ & $\begin{array}{l}+3.3-2.8 \\
+2.7-2.5 \\
+2.8-1.9 \\
+1.3-0.4 \\
+1.8-3.7\end{array}$ & $\begin{array}{l}1.95 \\
1.98 \\
1.51 \\
0.46 \\
1.70\end{array}$ \\
\hline \multicolumn{9}{|c|}{ Series $2-\mathrm{Fe}$ C, 30-g samples, tubes as received, vibrator and hammer } \\
\hline $\begin{array}{r}0 \text { to } 5 \\
5 \text { to } 10 \\
10 \text { to } 20 \\
+20 \\
\text { Recovery }\end{array}$ & $\begin{array}{l}6 \\
6 \\
6 \\
6 \\
6 \\
6\end{array}$ & $\begin{array}{l}9 \\
31 / 2 \\
21 / 4 \\
\end{array}$ & $\begin{array}{l}0.6 \\
2.4 \\
9.4\end{array}$ & $\begin{array}{l}14.5 \\
14.5 \\
14.5 \\
\end{array}$ & $\begin{array}{c}0.6 \\
2.4 \\
9.4 \\
\end{array}$ & $\begin{array}{r}32.97 \\
50.83 \\
15.12 \\
0.73 \\
99.62\end{array}$ & $\begin{array}{r}+1.2-0.8 \\
+1.2-1.0 \\
+0.8-0.3 \\
+.2-0.2 \\
+.4-0.4\end{array}$ & $\begin{array}{r}0.70 \\
.91 \\
.40 \\
.15 \\
.31\end{array}$ \\
\hline \multicolumn{9}{|c|}{ Series 3-Fe C, 30-g samples, tubes as received, vibrator and hammer } \\
\hline $\begin{array}{r}0 \text { to } 10 \\
10 \text { to } 20 \\
+20 \\
\text { Recovery } . . .\end{array}$ & $\begin{array}{l}4 \\
4 \\
4 \\
4 \\
4\end{array}$ & $\begin{array}{c}41 / 2 \\
21 / 4 \\
\\
\end{array}$ & $\begin{array}{c}2.4 \\
9.4 \\
\end{array}$ & $\begin{array}{l}14.5 \\
14.5 \\
\end{array}$ & $\begin{array}{c}2.4 \\
9.4 \\
\end{array}$ & $\begin{array}{r}79.90 \\
18.62 \\
0.82 \\
99.35\end{array}$ & $\begin{array}{r}+0.4-0.9 \\
+.9-0.4 \\
+.1-0.1 \\
+0 \quad-0.1\end{array}$ & $\begin{array}{r}0.53 \\
.52 \\
.08 \\
.05\end{array}$ \\
\hline \multicolumn{9}{|c|}{ Series 4 - Cu C, 15-g samples, tubes as received, vibrator and hammer } \\
\hline $\begin{array}{r}0 \text { to } 5 \\
5 \text { to } 10 \\
10 \text { to } 20 \\
+20 \\
\text { Recovery }\end{array}$ & $\begin{array}{l}5 \\
5 \\
5 \\
5 \\
5\end{array}$ & $\begin{array}{l}9 \\
41 / 2 \\
21 / 4\end{array}$ & $\begin{array}{c}0.67 \\
2.7 \\
10.7\end{array}$ & $\begin{array}{l}16.5 \\
16.5 \\
16.5 \\
\end{array}$ & $\begin{array}{c}0.67 \\
2.7 \\
10.7 \\
\end{array}$ & $\begin{array}{r}20.08 \\
38.91 \\
33.18 \\
5.68 \\
97.84\end{array}$ & $\begin{array}{r}+0.6-0.6 \\
+.9-0.8 \\
+.7-0.7 \\
+.3-0.3 \\
+.5-0.3\end{array}$ & $\begin{array}{r}0.42 \\
.59 \\
.50 \\
.19 \\
.28\end{array}$ \\
\hline
\end{tabular}

TABLE 7. Roller analyses of permalloy, PF, and atomized zinc, Zn, powders, 40-g samples, minus No. 325 sieve, 30-min intervals, 10-percent end point, dry air

\begin{tabular}{|c|c|c|c|c|c|c|c|c|c|c|c|}
\hline \multirow{2}{*}{$\begin{array}{c}\text { Fraction } \\
\text { (microns) }\end{array}$} & \multirow{2}{*}{$\begin{array}{c}\text { Number of } \\
\text { tests }\end{array}$} & \multirow{2}{*}{$\begin{array}{c}\text { Diameter of } \\
\text { settling } \\
\text { tube }\end{array}$} & \multirow{2}{*}{$\begin{array}{l}\text { Stokes air } \\
\text { velocity }\end{array}$} & \multirow{2}{*}{ Air flow } & \multirow{2}{*}{$\begin{array}{c}\text { Average air } \\
\text { velocity }\end{array}$} & \multicolumn{3}{|c|}{$\begin{array}{c}\text { Roller analysis-percentage by weight } \\
\text { of original samples }\end{array}$} & \multicolumn{3}{|c|}{$\begin{array}{l}\text { Typical microscopic count-- } \\
\text { percentage by weight of fraction }\end{array}$} \\
\hline & & & & & & Mean & $\begin{array}{l}\text { Maximum } \\
\text { deviation } \\
\text { from mean }\end{array}$ & $\begin{array}{l}\text { Standard } \\
\text { deviation }\end{array}$ & Undersize & Oversize & $\begin{array}{l}\text { Within size } \\
\text { limits }\end{array}$ \\
\hline \multicolumn{12}{|c|}{ Series 1-PF powder, tubes as received, vibrator and hammer } \\
\hline $\begin{array}{r}0 \text { to } 5 \\
5 \text { to } 10 \\
10 \text { to } 20 \\
+20 \\
\text { Recovery }\end{array}$ & $\begin{array}{l}5 \\
5 \\
5 \\
5 \\
5\end{array}$ & $\begin{array}{c}\text { Inches } \\
9 \\
41 / 2 \\
21 / 4 \\
\\
\end{array}$ & $\begin{array}{c}\mathrm{cm} / \mathrm{sec} \\
0.65 \\
2.6 \\
10.4 \\
\cdots\end{array}$ & $\begin{array}{c}L / \min \\
16.0 \\
16.0 \\
16.0 \\
\cdots\end{array}$ & $\begin{array}{l}\mathrm{cm} / \mathrm{sec} \\
0.65 \\
2.6 \\
10.4 \\
- \\
-\end{array}$ & $\begin{array}{r}40.40 \\
40.94 \\
16.54 \\
0.78 \\
98.52\end{array}$ & $\begin{array}{r}+1.0-1.0 \\
+1.2-1.1 \\
+0.4-0.5 \\
+\quad .2-0.2 \\
+\quad .2-0.4\end{array}$ & $\begin{array}{r}0.93 \\
.93 \\
.37 \\
.13 \\
.24\end{array}$ & (n. & (n) & (1) \\
\hline \multicolumn{12}{|c|}{ Series $2-Z n$ powder, small tubes lapped, vibrator and hammer, air velocity partly corrected a } \\
\hline $\begin{array}{r}0 \text { to } 5 \\
5 \text { to } 10 \\
10 \text { to } 20 \\
20 \text { to } 40 \\
+40 \\
\text { Recovery }\end{array}$ & $\begin{array}{rr}\text { b } & 10 \\
\text { b } 10 \\
\text { b } 10 \\
\\
\\
\\
\\
& 5 \\
& 5\end{array}$ & \begin{tabular}{l}
9 \\
$41 / 2$ \\
$21 / 4$ \\
$11 / 4$ \\
\\
\hdashline
\end{tabular} & $\begin{array}{c}0.53 \\
2.1 \\
8.5 \\
34.1 \\
\\
\end{array}$ & $\begin{array}{c}13.1 \\
13.1 \\
13.1 \\
13.1 \\
\cdots \\
\ldots . .\end{array}$ & $\begin{array}{c}0.53 \\
2.1 \\
8.5 \\
27.6 \\
\\
\end{array}$ & $\begin{array}{r}22.77 \\
34.06 \\
25.09 \\
15.50 \\
1.56 \\
98.88\end{array}$ & $\begin{array}{r}+0.3-0.5 \\
+1.0-1.1 \\
+0.6-0.8 \\
+\quad .3-0.2 \\
+\quad .1-0.1 \\
+\quad .7-0.7\end{array}$ & $\begin{array}{r}0.31 \\
.74 \\
.47 \\
.17 \\
.08 \\
.50\end{array}$ & $\begin{array}{l}0 \\
0.2 \\
.5 \\
.3 \\
.8 \\
\end{array}$ & $\begin{array}{l}0 \\
0.2 \\
7.4 \\
7.2 \\
0\end{array}$ & $\begin{array}{l}100 \\
99.6 \\
99.1 \\
92.5 \\
99.2 \\
-\end{array}$ \\
\hline \multicolumn{12}{|c|}{ Series $3-Z n$ powder, small tubes lapped, vibrator and hammer, air velocity corrected ( $V=K^{\prime \prime} \mathrm{d}^{t .4}$ above 20 microns) } \\
\hline $\begin{array}{r}20 \text { to } 30 \\
30 \text { to } 40 \\
+40 \\
\text { Recovery }\end{array}$ & $\begin{array}{l}5 \\
5 \\
5 \\
5\end{array}$ & $\begin{array}{l}11 / 2 \\
11 / 4 \\
\cdots\end{array}$ & $\begin{array}{c}19.2 \\
34.1 \\
- \\
\end{array}$ & $\begin{array}{l}10.9 \\
11.3 \\
\\
\end{array}$ & $\begin{array}{c}15.9 \\
23.8 \\
\cdots\end{array}$ & $\begin{array}{r}8.74 \\
4.20 \\
3.62 \\
98.58\end{array}$ & $\begin{array}{r}+0.3-0.3 \\
+\quad .7-0.5 \\
+\quad .4-0.4 \\
+\quad .3-0.3\end{array}$ & $\begin{array}{r}0.21 \\
.42 \\
.35 \\
.23\end{array}$ & $\begin{array}{c}0.4 \\
1.4 \\
2.0 \\
\end{array}$ & $\begin{array}{l}1.7 \\
0.6 \\
0\end{array}$ & $\begin{array}{c}97.9 \\
98.0 \\
98.0 \\
-\end{array}$ \\
\hline
\end{tabular}

a Air flow coresponding to Stokes air velocity used in all tubes except $1 \frac{1}{4} \mathrm{in}$. for which the air flow was calculated for $1 \frac{1 / 8}{8}$ inch diameter.

$\mathrm{b}$ These 10 tests include the 0 - to 5-, 5- to- 10-, and 10- to 20- micron fractions of series 2 and 3. 
TABLE 8. Roller analyses of unsieved permalloy, $P C$, and atomized copper, $\mathrm{Cu} A$, powders, with air velocity corrected $\left(V=K^{\prime \prime} d^{1.4}\right.$ above 20 microns), 10-percent end point, vibrator and hammer, small tubes lapped, dry air

\begin{tabular}{|c|c|c|c|c|c|c|c|c|c|c|c|}
\hline \multirow{2}{*}{$\begin{array}{c}\text { Fraction } \\
\text { (microns) }\end{array}$} & \multirow{2}{*}{$\begin{array}{l}\text { Number of } \\
\text { tests }\end{array}$} & \multirow{2}{*}{$\begin{array}{l}\text { Diameter of } \\
\text { settling } \\
\text { tube }\end{array}$} & \multirow{2}{*}{$\begin{array}{l}\text { Stokes air } \\
\text { velocity }\end{array}$} & \multirow{2}{*}{ Air flow } & \multirow{2}{*}{$\begin{array}{l}\text { Average air } \\
\text { velocity }\end{array}$} & \multicolumn{3}{|c|}{$\begin{array}{c}\text { Roller analysis-percentage by weight } \\
\text { of original samples }\end{array}$} & \multicolumn{3}{|c|}{$\begin{array}{l}\text { Typical microscopic count- } \\
\text { percentage by weight of fraction }\end{array}$} \\
\hline & & & & & & Mean & $\begin{array}{l}\text { Maximum } \\
\text { deviation } \\
\text { from mean }\end{array}$ & $\begin{array}{l}\text { Standard } \\
\text { deviation }\end{array}$ & Undersize & Oversize & $\begin{array}{c}\text { Within size } \\
\text { limits }\end{array}$ \\
\hline \multicolumn{12}{|c|}{ Series 1-PC powder, unsieved, 40-g samples } \\
\hline $\begin{array}{r}0 \text { to } 10 \\
10 \text { to } 20 \\
20 \text { to } 30 \\
30 \text { to } 40 \\
+40 \\
\text { Recovery }\end{array}$ & $\begin{array}{l}5 \\
5 \\
5 \\
5 \\
5 \\
5\end{array}$ & $\begin{array}{c}\text { Inches } \\
41 / 2 \\
21 / 4 \\
11 / 2 \\
11 / 4 \\
\end{array}$ & $\begin{array}{c}\mathrm{cm} / \mathrm{sec} \\
2.6 \\
10.4 \\
23.4 \\
41.6 \\
\end{array}$ & $\begin{array}{c}L / \min \\
16.0 \\
16.0 \\
12.9 \\
13.3 \\
-\end{array}$ & $\begin{array}{c}\mathrm{cm} / \mathrm{sec} \\
2.6 \\
10.4 \\
18.7 \\
27.9 \\
\end{array}$ & $\begin{array}{r}0.56 \\
52.60 \\
32.60 \\
9.74 \\
3.96 \\
99.46\end{array}$ & $\begin{array}{l}+0.1-0.1 \\
+.4-0.4 \\
+.3-0.6 \\
+.8-0.5 \\
+.1-0.2 \\
+.2-0.6\end{array}$ & $\begin{array}{l}0.08 \\
.28 \\
.35 \\
.49 \\
.10 \\
.29\end{array}$ & $\begin{array}{l}0 \\
0 \\
0.1 \\
.7 \\
.9 \\
\end{array}$ & $\begin{array}{l}0 \\
1.3 \\
1.5 \\
0.2 \\
0\end{array}$ & $\begin{array}{r}100 \\
98.7 \\
98.4 \\
99.1 \\
99.1 \\
-\end{array}$ \\
\hline \multicolumn{12}{|c|}{ Series 2-PC powder, unsieved, $100-g$ samples } \\
\hline $\begin{array}{r}0 \text { to } 20 \\
+20 \\
\text { Recovery }\end{array}$ & $\begin{array}{l}5 \\
5 \\
5\end{array}$ & $21 / 4$ & 10.4 & $\begin{array}{c}16.0 \\
-1\end{array}$ & 10.4 & $\begin{array}{l}50.00 \\
49.78 \\
99.78\end{array}$ & $\begin{array}{r}+0.8-0.6 \\
+\quad .7-0.8 \\
+\quad .1-0.2\end{array}$ & $\begin{array}{r}0.59 \\
.58 \\
.10\end{array}$ & $\begin{array}{c}0 \\
1.4 \\
-\end{array}$ & $\begin{array}{c}0 \\
0 \\
-\end{array}$ & $\begin{array}{c}100 \\
98.6\end{array}$ \\
\hline \multicolumn{12}{|c|}{ Series 3 -PC powder, accumulated +20 fractions, 40 -g samples } \\
\hline $\begin{array}{r}0 \text { to } 30 \\
30 \text { to } 40 \\
40 \text { to } 50 \\
50 \text { to } 60 \\
60 \text { to } 70 \\
+70 \\
\text { Recovery }\end{array}$ & $\begin{array}{l}1 \\
1 \\
1 \\
1 \\
1 \\
1 \\
1 \\
1\end{array}$ & $\begin{array}{l}11 / 4 \\
11 / 4 \\
11 / 4 \\
11 / 4 \\
11 / 4 \\
-\end{array}$ & $\begin{array}{r}23.4 \\
41.6 \\
65.0 \\
93.6 \\
127.4 \\
\end{array}$ & $\begin{array}{l}12.9 \\
13.3 \\
18.2 \\
23.4 \\
29.1 \\
\end{array}$ & $\begin{array}{l}18.7 \\
27.9 \\
38.2 \\
49.3 \\
61.2 \\
\end{array}$ & $\begin{array}{r}67.0 \\
25.6 \\
5.3 \\
1.3 \\
0.4 \\
.2 \\
99.8\end{array}$ & & ( & $\begin{array}{l}0 \\
3.3 \\
0.7 \\
.8 \\
2.5 \\
3.2 \\
\end{array}$ & $\begin{array}{l}0.4 \\
.7 \\
1.7 \\
0.5 \\
2.9 \\
0\end{array}$ & $\begin{array}{l}99.6 \\
96.0 \\
97.6 \\
98.7 \\
94.6 \\
98.5\end{array}$ \\
\hline \multicolumn{12}{|c|}{ Series 4-Cu A powder, unsieved, 40-g samples } \\
\hline $\begin{array}{l}0 \text { to } 30 \\
30 \text { to } 40 \\
40 \text { to } 50 \\
50 \text { to } 60 \\
60 \text { to } 70 \\
70 \text { to } 80\end{array}$ & $\begin{array}{l}5 \\
5 \\
5 \\
5 \\
5 \\
5\end{array}$ & $\begin{array}{l}11 / 2 \\
11 / 4 \\
11 / 4 \\
1114 \\
11 / 4 \\
11 / 4\end{array}$ & $\begin{array}{r}24.1 \\
42.9 \\
67.0 \\
96.5 \\
131.3 \\
171.5\end{array}$ & $\begin{array}{l}13.1 \\
13.6 \\
18.6 \\
24.0 \\
29.7 \\
35.9\end{array}$ & $\begin{array}{l}19.1 \\
28.6 \\
39.1 \\
50.5 \\
62.6 \\
75.5\end{array}$ & $\begin{array}{r}21.62 \\
10.98 \\
11.02 \\
9.66 \\
11.80 \\
7.02\end{array}$ & $\begin{array}{l}+1.1-0.8 \\
+0.5-1.0 \\
+1.3-0.9 \\
+0.5-0.4 \\
+\quad .5-0.9 \\
+\quad .4-0.4\end{array}$ & $\begin{array}{l}0.75 \\
.54 \\
.76 \\
.38 \\
.48 \\
.34\end{array}$ & $\begin{array}{l}0 \\
0.1 \\
.9 \\
3.2 \\
3.1 \\
2.7\end{array}$ & $\begin{array}{r}0.3 \\
2.7 \\
2.3 \\
2.1 \\
2.1 \\
12.5\end{array}$ & $\begin{array}{l}99.7 \\
97.2 \\
96.8 \\
94.7 \\
94.8 \\
84.8\end{array}$ \\
\hline $\begin{array}{r}80 \text { to } 90 \\
90 \text { to } 100 \\
100 \text { to } 110 \\
110 \text { to } 120 \\
+120 \\
\text { Recovery }\end{array}$ & $\begin{array}{l}5 \\
5 \\
5 \\
5 \\
5 \\
5\end{array}$ & $\begin{array}{l}11 / 4 \\
11 / 4 \\
11 / 4 \\
11 / 4 \\
\\
\end{array}$ & $\begin{array}{l}217.1 \\
268.0 \\
324.3 \\
385.9 \\
\end{array}$ & $\begin{array}{c}42.3 \\
49.0 \\
56.0 \\
63.2 \\
- \\
\end{array}$ & $\begin{array}{r}89.1 \\
103.2 \\
117.9 \\
133.0 \\
\\
\end{array}$ & $\begin{array}{r}6.76 \\
5.70 \\
4.92 \\
4.18 \\
5.14 \\
99.10\end{array}$ & $\begin{array}{l}+.4-0.5 \\
+\quad .3-0.4 \\
+\quad .7-0.4 \\
+\quad .6-0.8 \\
+\quad .4-0.5 \\
+\quad .3-0.4\end{array}$ & $\begin{array}{l}.32 \\
.28 \\
.39 \\
.57 \\
.32 \\
.28\end{array}$ & $\begin{array}{r}4.0 \\
9.6 \\
7.1 \\
11.5 \\
12.1 \\
\end{array}$ & $\begin{array}{c}14.8 \\
14.3 \\
16.9 \\
22.1 \\
0\end{array}$ & $\begin{array}{l}81.2 \\
76.1 \\
76.0 \\
66.4 \\
87.9 \\
\end{array}$ \\
\hline
\end{tabular}


TABLE 9. Roller analyses of accumulated Roller fractions of permalloy mix, $P M$, powder, with air velocity corrected $\left(V=K^{\prime \prime} d^{1.4}\right.$ above 20 microns) 10-percent end point, vibrator and hammer, small tubes lapped, dry air

\begin{tabular}{|c|c|c|c|c|c|c|c|}
\hline \multirow{2}{*}{$\begin{array}{c}\text { Fraction } \\
\text { (microns) }\end{array}$} & \multirow{2}{*}{$\begin{array}{l}\text { Number } \\
\text { of tests }\end{array}$} & \multirow{2}{*}{$\begin{array}{l}\text { Diameter } \\
\text { of settling } \\
\text { tube }\end{array}$} & \multirow{2}{*}{$\begin{array}{l}\text { Stokes air } \\
\text { velocity }\end{array}$} & \multirow{2}{*}{ Air flow } & \multirow{2}{*}{$\begin{array}{l}\text { A verage } \\
\text { air } \\
\text { velocity }\end{array}$} & \multicolumn{2}{|c|}{$\begin{array}{l}\text { Roller analysis-per- } \\
\text { centage by weight of } \\
\text { original sample }\end{array}$} \\
\hline & & & & & & Mean & $\begin{array}{l}\text { Maximum } \\
\text { deviation } \\
\text { from mean }\end{array}$ \\
\hline \multicolumn{8}{|c|}{ Series 1 -A ccumulated 0 to 10 fraction, $40-\mathrm{g}$ samples } \\
\hline $\begin{array}{r}0 \text { to } 10 \\
10 \text { to } 20 \\
20 \text { to } 30 \\
30 \text { to } 40 \\
+40 \\
\text { Recovery }\end{array}$ & $\begin{array}{l}2 \\
2 \\
2 \\
2 \\
2 \\
2\end{array}$ & $\begin{array}{c}\text { Inches } \\
41,2 \\
21 / 4 \\
11 / 2 \\
11 / 4 \\
\end{array}$ & $\begin{array}{c}\mathrm{cm} / \mathrm{sec} \\
2.6 \\
10.4 \\
23.4 \\
41.6 \\
\\
\end{array}$ & $\begin{array}{c}L / \min \\
16.0 \\
16.0 \\
12.9 \\
13.3 \\
\end{array}$ & $\begin{array}{c}\mathrm{cm} / \mathrm{sec} \\
2.6 \\
10.4 \\
18.7 \\
27.9 \\
\end{array}$ & $\begin{array}{r}81.6 \\
15.8 \\
1.0 \\
0.2 \\
0.1 \\
98.6\end{array}$ & $\begin{array}{l}+0.5-0.6 \\
+.1-0 \\
+.1-0.2 \\
+0-0.1 \\
+0-0 \\
+0.6-0.7\end{array}$ \\
\hline \multicolumn{8}{|c|}{ Series 2-Accumulated 10-20 fraction, 40-g samples } \\
\hline $\begin{array}{r}0 \text { to } 10 \\
10 \text { to } 20 \\
20 \text { to } 30 \\
30 \text { to } 40 \\
+40 \\
\text { Recovery }\end{array}$ & $\begin{array}{l}2 \\
2 \\
2 \\
2 \\
2 \\
2\end{array}$ & $\begin{array}{l}41 / 2 \\
21 / 4 \\
11 / 2 \\
11 / 4 \\
\cdots\end{array}$ & $\begin{array}{r}2.6 \\
10.4 \\
23.4 \\
41.6 \\
\end{array}$ & $\begin{array}{l}16.0 \\
16.0 \\
12.9 \\
13.3 \\
\end{array}$ & $\begin{array}{r}2.6 \\
10.4 \\
18.7 \\
27.9 \\
\end{array}$ & $\begin{array}{r}1.6 \\
96.3 \\
1.0 \\
0.4 \\
0.1 \\
99.3\end{array}$ & $\begin{array}{r}+0.4-0.5 \\
+.1-0.1 \\
+.4-0.5 \\
+.3-0.2 \\
+.1-0.1 \\
+.6-0.6\end{array}$ \\
\hline \multicolumn{8}{|c|}{ Series 3 -A ccumulated 20 to 30 fraction, 25-g sample } \\
\hline $\begin{array}{r}0 \text { to } 10 \\
10 \text { to } 20 \\
20 \text { to } 30 \\
30 \text { to } 40 \\
+40 \\
\text { Recovery }\end{array}$ & $\begin{array}{l}1 \\
1 \\
1 \\
1 \\
1 \\
1 \\
1\end{array}$ & $\begin{array}{l}41 / 2 \\
21 / 4 \\
11 / 2 \\
11 / 4 \\
-\end{array}$ & $\begin{array}{r}2.6 \\
10.4 \\
23.4 \\
41.6\end{array}$ & $\begin{array}{l}16.0 \\
16.0 \\
12.9 \\
13.3 \\
\end{array}$ & $\begin{array}{r}2.6 \\
10.4 \\
18.7 \\
27.9 \\
\end{array}$ & $\begin{array}{r}0 \\
17.6 \\
76.7 \\
5.1 \\
0.1 \\
99.5\end{array}$ & - \\
\hline \multicolumn{8}{|c|}{ Series 4-A ccumulated 30 to 40 fraction, 8-g sample } \\
\hline $\begin{array}{r}0 \text { to } 10 \\
10 \text { to } 20 \\
20 \text { to } 30 \\
30 \text { to } 40 \\
+40 \\
\text { Recovery }\end{array}$ & $\begin{array}{l}1 \\
1 \\
1 \\
1 \\
1 \\
1 \\
1\end{array}$ & $\begin{array}{l}41 / 2 \\
21 / 4 \\
11 / 2 \\
11 / 4 \\
\cdots\end{array}$ & $\begin{array}{r}2.6 \\
10.4 \\
23.4 \\
41.6 \\
\end{array}$ & $\begin{array}{l}16.0 \\
16.0 \\
12.9 \\
13.3 \\
\\
\end{array}$ & $\begin{array}{r}2.6 \\
10.4 \\
18.7 \\
27.9 \\
\end{array}$ & $\begin{array}{r}0.3 \\
0.4 \\
17.9 \\
76.1 \\
5.4 \\
100.1\end{array}$ & (n) \\
\hline \multicolumn{8}{|c|}{ Series 5 -Accumulated +40 fraction, $2.82-\mathrm{g}$ sample } \\
\hline $\begin{array}{r}0 \text { to } 10 \\
10 \text { to } 20 \\
20 \text { to } 30 \\
30 \text { to } 40 \\
+40 \\
\text { Recovery }\end{array}$ & $\begin{array}{l}1 \\
1 \\
1 \\
1 \\
1 \\
1 \\
1\end{array}$ & $\begin{array}{l}41 / 2 \\
21 / 4 \\
11 / 2 \\
11 / 4 \\
-\end{array}$ & $\begin{array}{r}2.6 \\
10.4 \\
23.4 \\
41.6 \\
\end{array}$ & $\begin{array}{l}16.0 \\
16.0 \\
12.9 \\
13.3 \\
\end{array}$ & $\begin{array}{r}2.6 \\
10.4 \\
18.7 \\
27.9 \\
\end{array}$ & $\begin{array}{r}0.4 \\
0.4 \\
0.4 \\
20.2 \\
79.1 \\
100.5\end{array}$ & (n) \\
\hline
\end{tabular}




\subsection{Condition of Interior Surface of Settling Tube}

The tabulated results show that for diameters up to 20 microns, good reproducibility (on the order of \pm 1 percent of the original weight of the sample) was obtained with powders such as $\mathrm{Cu} \mathrm{A}, \mathrm{Cu} B, \mathrm{Fe} \mathrm{A}$, $\mathrm{Fe} \mathrm{B} 1$, and $\mathrm{Fe} \mathrm{B} 2$, which contained relatively small amounts (5 percent or less) of the 0 - to 5-micron fraction (tables $3,4,5$ ). Good results were also obtained with the PM powder (0- to 5-micron fraction, theoretically, about 13.5 percent) without the use of a vibrator if the initial separation was made at 10 microns (series 3, table 2). However, for separations made at 30 or 40 microns in the $1 \frac{1 / 2}{\text { - }}$ and $1 \frac{1}{4}$-in. settling tubes as received, the results often varied widely. These variations were attributed to the rough condition of the interior surface of some of these tubes. The 9-, 41/2-, and 21/4-in. tubes were made of welded stainless steel sheet, and the interior surfaces were smooth. The original 11/4-in. tubes supplied with the unit were made of brass tubing and failed after relatively short periods of service. One of these tubes was used only in series 1 of table 4 , and gave reproducible results; another was used only for series 1 of table 5 and gave variable results. Whether or not these inconsistencies were due to the condition of the interior surfaces of these tubes cannot be stated, because they were not examined upon removal prior to returning them for replacement.

In all the other tabulated series of tests requiring

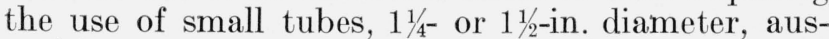
tenitic stainless steel tubes were employed. Both of these tubes were made of stainless-steel tubing, machined to the required inside diameter, and the interior surfaces were relatively rough. Powder particles adhering to these rough surfaces were difficult to dislodge, and substantial amounts of undersized material were often carried over into the next fraction. In series 3 of table 2 , for example, microscopic measurements of the 30 - to 40-micron fraction showed the presence of 24.8 percent of undersize material, which had obviously remained as a residue from the prior 30 micron separation. Such condition caused wide variations in the results obtained for both the 20 - to 30 - and 30 - to 40 -micron fractions. The carryover of undersized material was considerably reduced with consequent improvement in reproducibility when a vibrator was used in addition to the regular hammer (series 4, table 2). Still further improvement in this respect was observed when the 30 -micron separation was made in the smooth $2 \frac{1}{4}$-in. tube (series 5 , table 2 ), or when the interior surfaces of the smaller tubes were lapped (series 6 , table 2). The amount of oversized material was reduced materially only by lowering the air velocity (series 7 , table 2). Reproducible results were obtained with all other powders tested with these tubes when the interior surfaces were lapped (tables $3,4,5,7$, and 8 ).

\subsection{Dislodgment of Small Particles}

Efficient separations at the 5-micron level of powders such as $\mathrm{PF}, \mathrm{PM}, \mathrm{Fe} \mathrm{C}, \mathrm{Cu} \mathrm{C}$, and $\mathrm{Zn}$, which contain substantial amounts (13.5 to 40.4 percent) of 0 - to 5-micron fraction, were found to be difficult because of the tendency of particles to adhere to the walls of the settling tube. In such cases the hammer device alone was not sufficiently effective in dislodging the particles, consequently the results were variable and the total recovery was low (series 1 , tables 2 and 6 ). But when a vibrator was used in addition to the regular hammer device, variations in results were diminished and the total recovery was markedly improved (tables 2, 6, and 7).

\subsection{Size Range of Fraction}

It will be noted from the tables that a separation at a larger particle diameter usually gave somewhat lower results than the accumulated weights of the finer fractions. In series 2 of tables 2 and 3 , for example, the average for the 0 - to 20 -micron fractions of $\mathrm{PM}$ and $\mathrm{Cu} \mathrm{B}$ powders was 68.9 and 49.6 percent, respectively, whereas the sums of the finer fractions $(0-$ to 10 - and 10 - to 20 -micron in series 3 , table 2 , and 0 to 5,5 to 10 , and 10 to 20 in series 1 , table 3) were 70.8 and 53.4, respectively. This is believed to be a consequence of the uncertainty of the end point under some conditions. For the 0- to 20-micron fractions, the rate of separation for the initial 30-min intervals was very high (as much as 60 percent by weight of sample). However, this rate fell off rapidly, and at the 10 -percent end point as much as 4 or 5 percent was still being blown over per interval. This clearly indicates that some of the border-line particles were not being separated.

Similar end-point uncertainty was found when accumulated, sized, fractions were analyzed by the Roller apparatus, as shown in table 9 . The samples in these tests were the accumulated fractions of the 5 tests included in series 7 of table 2. These fractions contain many border-line particles that may be blown over or remain in the settling tubes, depending on the number of such particles present and the rate of separation. A sample of accumulated 20 - to 30-micron fractions, for example, may contain some 18 and 19 microns as well as 31, 32, and possibly a few 33-micron particles. If a separation is made at 20 microns, the initial rate of separation will be low but will fall off slowly, so that by the time the end point is reached all of the undersize, most of the 20-micron, and many 21 - and 22-micron particles will have been blown over. This may result in the separation of a nominal minus 20 -micron fraction of appreciable size, even though repeated microscopic measurements have indicated that 98 to 99 percent by weight of the original sample was within the 20- to 30 -micron size limits. When the separation is made at 30 microns, however, the initial rate of separation will be very high and will fall off rapidly, so that some of the border-line particles will remain in the tube to be blown over during the 40-micron separation. 


\subsection{Size of Samples}

The size of sample, within the relatively small limits imposed by the diameter of the sample tube (M, fig. 1), appeared to have little effect on Roller particle-size analyses. This has been indicated previously by the inclusion of tests ( 3 for each weight) of samples, weighing $20,30,40,60,80$, and $100 \mathrm{~g}$, in the results represented by figure 6 . It was noted, however, that the results for the 80 - and 100-g samples were near the lower limit of the range, and large numbers of such tests might broaden the range. On the other hand, with very small samples, particularly of fine powders, the small losses of material unavoidably obtained become increasingly important on a percentage basis (see total recovery values of tables 2 , 6,7 , and 8). In this investigation, consistent results were obtained by following the suggestion of the Metal Disintegrating Co. [9] in using samples weighing about 10 times the numerical value of the apparent density of the powder, with a maximum of $40 \mathrm{~g}$ in most cases.

\subsection{Humidity of Air}

The tendency of small particles to stick to each other or to the walls of the settling tubes was found to be markedly affected, in some cases, by the humidity of the air supplied to the settling tubes. The solid curves, E, A, and B shown in figure 8 represent the range of results obtained during the separation of the 0 - to 10 - or 0 - to 5-micron fractions of the PM, $\mathrm{Fe} \mathrm{C}$, and $\mathrm{Cu} \mathrm{B}$ powders with dried air and with hammer only. The broken curves $\mathrm{E}^{\prime}, \mathrm{A}^{\prime}$, and $\mathrm{B}^{\prime}$ represent the results obtained with humidified air and with hammer only. The air was humidified by passing it, in two stages, through alundum thimbles immersed in large bottles of water. The relative humidity, as measured by wet- and dry-bulb thermometry, was about 90 percent.

Curves $\mathrm{E}$ and $\mathrm{E}^{\prime}$ of figure 8 show that high humidity greatly increased the separation rate of the 0 - to 10-micron fraction of the PM powder. The final results, however, were within the range of those obtained with dried air. 'The effect of humidity on the initial rate of separation of the 0 - to 5 -micron fraction of the $\mathrm{Cu} \mathrm{B}$ was negligible, but the final results were considerably higher than those obtained with dried air (curves $B$ and $B^{\prime}$ ). Humidity had little effect on carbonyl iron powder, the results at all stages being within the range of those obtained with dried air (curves $\mathrm{A}$ and $\mathrm{A}^{\prime}$ ). With this powder, and with $\mathrm{Cu} \mathrm{C}$ powder, more efficient dislodgment of particles was obtained by the use of a vibrator in addition to the hammer as shown by curves $\mathrm{C}$ and $\mathrm{D}$ (see also tables $2,6,7$, and 8 ).

It is probable that the varying effect of high humidity may be connected with the static charges picked up by the particles during separation. These charges may vary in nature and magnitude with the humidity of the air, as well as with the size and composition of the particle.

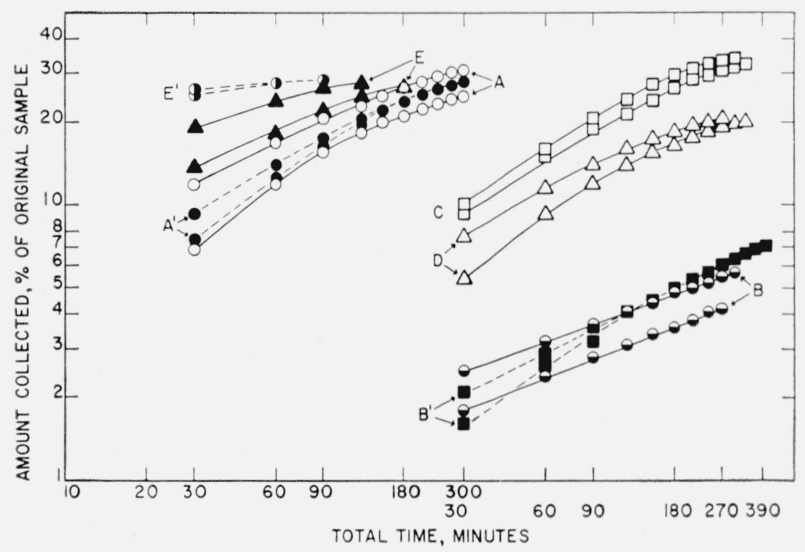

FIGURE 8. Effect of the humidity of the air stream on the separation of fine fractions of metal powders.

Curve E, 0- to 10-micron fraction of permalloy-mix powder obtained with dried air without vibrator; $\mathrm{E}^{\prime}$, same conditions except for relative humidity of the airair without vibrator; $\mathrm{E}^{\prime}$, same conditions except for relative humidity of the air-
about 90 percent; $\mathrm{A}, 0$ - to 5 -micron fraction of carbonyl iron, $\mathrm{Fe} \mathrm{C}$, powder obabout 90 percent; $\mathrm{A}$, 0 - to 5 -micron fraction of carbonyl iron, $\mathrm{Fe} \mathrm{C}$, powder ob-
tained with dried air without vibrator; $\mathrm{A}^{\prime}$, same except for relative humidity of the air-about 90 percent; $\mathrm{B}, 0$ - to 5 -micron fraction of electrolytic copper, $\mathrm{Cu}$ B, powder obtained with dried air without vibrator; $\mathrm{B}^{\prime}$, same except for relative humidity of the air-about 90 percent; C, 0 - to 5-micron fraction of carbonyl iron, $\mathrm{Fe} \mathrm{C}$, powder obtained with dry air and with vibrator and hammer; $\mathrm{D}, 0$ - to 5-micron fraction of electrolytic copper, $\mathrm{Cu} \mathrm{C}$, powder obtained with dry air and with a vibrator in addition to the regular hammer.

\subsection{Particle Shape}

With the metal powders used in these experiments, particle shape was found to have no effect on the Roller analyses insofar as reproducibility was concerned. With the modifications in equipment previously discussed, and with reasonably uniform operating conditions, reproducible results were obtained with all powders tested at air velocities calculated for diameters of 5 to 40 microns. However, the accuracy of these separations, or rather the accuracy of the values assigned to the size limits of the fractions, depends on the relation that is assumed to exist between the diameter of the particle and its falling velocity. The accuracy of some of these assumptions will be discussed in the next section.

\section{Accuracy of Separations}

The accuracy of Roller analyses was checked by microscopic measurements on sized fractions of permalloy, atomized copper, and atomized zinc powders. Samples of these powders, dispersed in xylene containing a few drops of cedar oil on plain glass slides, were mounted on a microscope; the images were projected with transmitted light upon a screen at 1,000 magnifications; and measured to the nearest millimeter. While it is recognized that there are inherent errors in this method of measurement, they are minute with respect to the present application of the method and, therefore, may be overlooked.

Microscopic measurements indicated that separations of these nearly spherical particles closely approximated the requirements of Stokes law for diameters of 5 to 20 microns. The parabolic curve A 


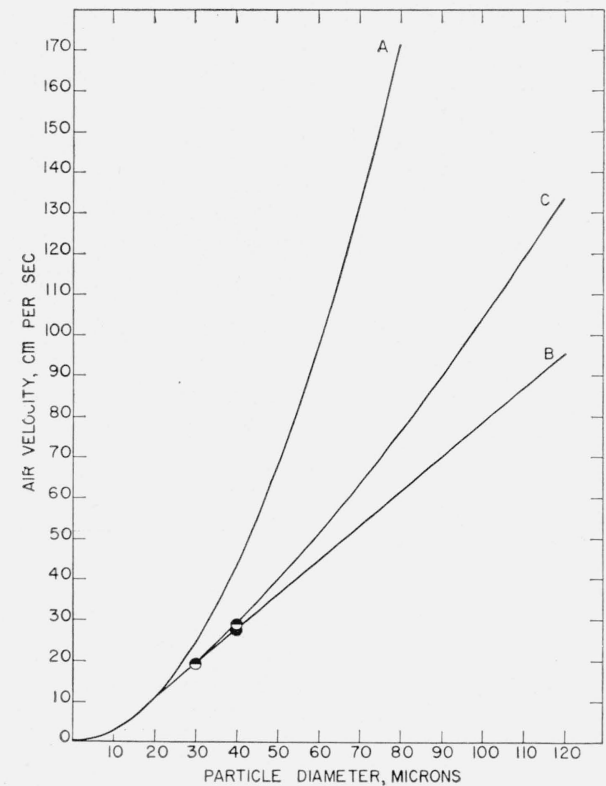

Figure 9. Curves showing theoretical relationships between air velocity and diameter of particle.

Curve A, $V=K d^{2} ; \mathrm{B}, V=C+K^{\prime} d ; \mathrm{C}, V=K^{\prime \prime} d^{1.4}$. Symbols $\odot$ and $\mathrm{represent}$ experimentally determined values.

of figure 9 , for example, shows the theoretical values for the terminal falling velocities of spherical PM particles, as calculated by Stokes law, plotted against the particle diameter in microns. In actual separations at air velocities taken from this curve, it was found that a minimum of 98.5 percent by weight of the 0 - to 10 - and 10 - to 20 -micron fractions were within the designated size limits, as shown by the microscopic counts of series 3 of table 2 . Similar results were obtained with 0 - to 5-, 0 - to $10-, 0$ - to $20-, 5$ to $10-$, and $10-$ to 20 -micron fractions of $\mathrm{Cu} \mathrm{A}, \mathrm{PF}$, $\mathrm{Zn}$, and $\mathrm{PC}$ powders, as shown in tables 3,7 , and 8 .

For separations at larger diameters, air velocities calculated by Stokes law were too high. For example, the air velocities required for separations of PM powder at 30 and 40 microns would be 23.4 and $41.6 \mathrm{~cm} / \mathrm{sec}$, respectively (see curve A, fig. 9). It was found, mostly by trial and error, that accurate separations of this powder could be obtained with air velocities of $18.7 \mathrm{~cm} / \mathrm{sec}$ for 30 microns, and either 27 or $28 \mathrm{~cm} / \mathrm{sec}$ for 40 microns.

Dallevalle [12], citing the work of Allen [13] and Martin [14], states that, between the region of streamline motion, where $V$ varies as the square of the particle diameter, and the region of fully turbulent motion, where $V$ varies as the square root of the particle diameter, there is a rather extensive region where a straight line relationship exists and $V$ varies as the first power of the particle diameter.

Curve $\mathrm{B}$ of figure 9 is a straight line through the points 18.7 and 27 determined experimentally as suitable for accurate separations at 30 and 40 microns respectively. This line cuts the parabola of Stokes law at about 20 microns. Tests were made of PC powder, using air velocities taken from curve $B$ for separations at diameters ranging by $10-\mu$ increments from 40 to 90 microns $(40,50,60$, etc.). These velocities were found to be too low for accurate separations. The nominal 70 - to 80 -micron fraction, for example, when measured microscopically actually was 100 percent within the 60 - to 70-micron range. Microscopic counts were made of each of the fractions to determine approximately the actual separation points. These points, when plotted on logarithmic coordinates against the air velocities used, fell near a straight line with a slope of approximately 1.4 .

This suggests a relationship similar to that used by Burke and Plummer [15], who related their own and other available data to the general formula for the resistance to motion, $R$, of a particle in a fluid $R=k \rho_{0}{ }^{n-1} \mu^{2-n}(d / 2)^{n} V^{n}$. The force causing motion is for a sphere, $F=\left(\pi d^{3} / 6\right)\left(\rho-\rho_{0}\right) g$. At terminal velocity $R=F$ and

$$
V^{n}=\frac{(2)^{n} \pi g}{6 k}\left(\rho-\rho_{0}\right) \rho_{0}{ }^{1-n} \mu^{n-2} d^{3-n} .
$$

When $n=1$, this equation becomes Stokes law for streamline motion, and when $n=2$, it becomes Newton's law for turbulent motion.

Burke and Plummer suggest that in the intermediate region $1<n<2$, and probably changes smootbly and continuously between the two limiting values.

If $n$ is made equal to 1.25 , eq (2) becomes

$$
V=2\left[\frac{\pi g\left(\rho-\rho_{0}\right)}{6 k}\right]^{0.8} \rho_{0}^{-0.2} \mu^{-0.6} d^{1.4} .
$$

With $k=16.07$, this gives for permalloy powder $V=18.7$ at 30 microns and $V=27.9$ at 40 microns. A theoretical curve for PM powder with air velocities calculated by this formula is shown as curve $\mathrm{C}$ in figure 9.

Tests were made on unsieved $\mathrm{PC}$ and $\mathrm{Cu} \mathrm{A}$ powders with air velocities calculated by eq (3) for particle diameters larger than 20 microns. The results shown in table 8 were comparable to microscopic measurements for particle diameters up to 70 microns, a minimum of 94.6 percent by weight of the 60 - to 70 -micron fraction being within the specified size limits. These results indicate that, for particles larger than those which obey Stokes law, the exponent $n$ of eq (2) changes very rapidly from a value of 1 at 20 microns to about 1.25 at 30 microns. Between 30 and 70 microns the change in $n$, if any, is too small to be detected by this method of testing. Above 70 microns the separations became progressively less sharp. There appeared to be some preponderance of oversized material in the larger fractions that may indicate that $n$ changes in this region to a value larger than 1.25. However, an increasing overlapping of size range among consecutive fractions (both undersize and oversize) made it impossible to draw definite conclusions. In spite of this overlapping, the results continued to be reproducible up to 120 microns, as may be seen in series 4 of table 8 . 
As previously stated, no attempt was made to check the Roller analyses of powders composed of irregularly shaped particles by microscopic measurements. Some work has been done in this field, but at the present time, the exact nature of the dimensions that control the measurement of an irregularly shaped particle by air elutriation is not well understood nor is the possible relationship of these dimensions to any visible dimensions or to the surface area or volume of the particle.

\section{Summary}

Subsieve particle size analyses of a number of metal powders were made with a Roller air analyzer, using 30-min. intervals and a 10-percent end-point criterion. This procedure was found to be satisfactory for samples of normal size distribution. The end point was somewhat uncertain, however, when (1) the initial rates were very low but persistent, as in a separation near the lower limit of an accululated, sized fraction, and (2) the initial rates were very high but fell off rapidly, as in a separation near the upper limit of an accumulated sized fraction, or with a relatively large range of sizes within the fraction such as 0 to 30 microns.

Particle shape appeared to have no effect on the reproducibility of the tests. Results reproducible within approximately \pm 1 percent by weight of sample were obtained with very irregularly shaped particles for diameters up to 40 microns, and with spherical particles for diameters up to 120 microns provided the following conditions were fulfilled:

1. An alundum extraction thimble was used to collect the fractions.

2. The inside surfaces of the settling tubes were smooth.

3. A Syntron vibrator was used in addition to the regular hammer, particularly for powders containing relatively large amounts of the 0 - to 5-micron fraction.

4. The size of the sample, while not cirtical, was maintained at approximately 10 times the apparent density as a lower limit, with $40 \mathrm{~g}$ as a maximum in most cases.

The effect of high humidity on the separation of metal powders was not consistent. With some powders the rate of separation was markedly increased, but with others no effect was noted. In view of this uncertainty, it would seem advisable to use dried air or air of relatively constant humidity for all tests.

The accuracy of analyses of spherical powders was checked by microscopic measurements of the frac- tions. With these powders Stokes law applied for diameters of 5 to 20 microns, and the air velocity could be calculated accurately as proportional to the square of the particle diameter $\left(V=K d^{2}\right)$. For larger diameters, the air velocity was found to be proportional to the 1.4 power of the diameter $\left(V=K^{\prime \prime} d^{1.4}\right)$. At still larger diameters, increased overlapping of size range among consecutive fractions prevented any definite conclusions, except that the results continued to be reproducible.

Grateful acknowledgment is made to W. Earl Lindlief, formerly of the National Bureau of Standards, who assisted in the planning and supervision of the early part of the investigation; and to John C. Everts and Caroline Plummer Jeffries for their patient and efficient assistance in the monotonous and time-consuming tasks of making the vitally important particle-size counts and analyses.

\section{References}

[1] P. S. Roller, Separation and size distribution of microscopic particles - an air analyzer for fine powders, U. S. Bureau of Mines Tech. Pap. No. 490 (1931).

[2] P. S. Roller, U. S. Patent 2019507 (1935)

[3] P. S. Roller, Measurement of particle size with an accurate air analyzer, ASTM Proc. 32 (II) 607 (1932).

[4] P. S. Roller, Size distribution of ceramic powders as determined by a particle size analyzer, J. Am. Ceram. Soc. 20, 167 (1951).

[5] MPA Standard 12-51T, Subsieve analysis of granular metal powders by air classification (1951).

[6] ASTM Tentative method for sub-sieve analysis of granular metal powders by air classification (under consideration) (1952).

[7] R. A. Castleman, Resistance to the steady motion of small spheres in fluids, NACA Technical Note 231 (1926).

[8] H. Wadell, New sedimentation formulas, Physies 5, 281 (1934).

[9] Metals Disentegrating Co., Elizabeth, N. J., Particle size analysis of metal powders (1946)

[10] P. S. Roller and P. V. Roundy, Surface area of portland cement, ASTM symposium on particle size measurement (1941)

[11] P. S. Roller, Metal powder size determination by Roller air analyzer, ASTM symposium on powder metallurgy (1952)

[12] J. M. Dallevalle, Micromerities (Pitman Publishing Corp., New York, N. Y., 1943).

[13] H. S. Allen, The motion of spheres in a viscous fluid, Phil. Mag. [5] 50, 323 (1900).

[14] G. Martin, On the diameters of irregularly shaped crushed sand particles lifted by air currents of different. speeds and different temperatures, Trans. Brit. Ceram. Soc. 26, 21 (1926)

[15] S. P. Burke and W. B. Plummer, Suspension of macroscopic particles in a turbulent gas stream, Ind. Eng. Chem. 20, 1196 (1928).

Washington, December 12, 1952 . 